\title{
Flame Synthesis of Functional Nanostructured Materials \& Devices: Surface Growth \& Aggregation
}

\author{
Georgios A. Kelesidis, Eirini Goudeli and Sotiris E. Pratsinis*
}

Particle Technology Laboratory, Institute of Process Engineering, Department of Mechanical and Process Engineering, ETH Zürich, Sonneggstrasse 3, CH-8092 Zürich, Switzerland.

$$
\text { Ph. +41 (0) } 4463231 \text { 80; Fax. +41 (0) } 446321595
$$

Submitted to:

Proceedings of the Combustion Institute

August 18, 2016

*Corresponding author: sotiris.pratsinis@ptl.mavt.ethz.ch 


\begin{abstract}
Combustion is essential to the manufacture of carbon black, fumed oxides, optical fibers and, recently, new high-value products like carbon nanotubes, nanosilver and biomagnetic nanofluids that are driven to market predominantly by startups. This technology is attractive for material synthesis for its proven scalability as it does not involve the tedious steps of wet chemistry and can readily form stably metastable compositions and high purity products.

Recent advances in aerosol and combustion sciences reveal that coagulation and sintering and/or surface growth control product particle size and morphology through the high temperature particle residence time, self-preserving size distribution and power laws for fractal-like particles. This motivates synthesis of an array of unique particle compositions and morphologies primarily by spray combustion leading to new catalysts, gas sensors and bio-materials and, most recently, to hand-held devices such as breath analysis sensors for monitoring chronic illnesses. In particular, multi-scale process design integrating mesoscale and molecular dynamics facilitates understanding of combustion product development.

The latter contributes also to understanding of aggregation and surface growth of nascent soot, a bona fide nanostructured material! So here nascent soot dynamics, after nucleation or inception, are investigated through accounting of soot agglomeration and surface growth by acetylene pyrolysis. Neglecting the fractal-like nature of soot underestimates its mobility diameter and polydispersity up to $40 \%$. The evolution of nascent soot structure from spheres to aggregates is quantified by the mass fractal dimension and mass-mobility exponent, in excellent agreement with microscopic and mass-mobility measurements in a standard burner-stabilized stagnation ethylene flame. Surface growth chemically bonds the constituent primary particles of these aggregates, while the effect of soot volume fraction on soot morphology is elucidated. Based on aggregate projected area, a scaling law is derived for determining the primary particle size of nascent soot aggregates from mass-mobility measurements rather than tedious image counting.
\end{abstract}

Keywords: flame aerosol synthesis; functional nanomaterials \& devices; coagulation and surface growth; aggregates and agglomerates; fractal dimension and mass-mobility exponent 


\section{Nomenclature}

$A$
$a$
$a_{a}$
$D_{a}$
$D_{f}$
$D_{f m}$
$D_{s}$
$d_{g}$
$d_{m}$
$d_{p}=6 v / a$
$D_{p f}=\left(4.6-D_{f}\right) / 1.628$
$f_{v}$
$k_{B}$
$K n_{D, m}$
$k_{a}$
$k_{f}$
$k_{f m}$
$k_{s}$
$m$
$m_{2 c}$
$N$
$N_{a v}$
$n$
$n_{p}=v / v_{p}$
$r$
$T$
$t$
$v$

\section{Greek letters}$$
\beta
$$$$
\gamma
$$$$
\theta
$$$$
\rho_{s}
$$$$
\sigma_{g, m}
$$$$
\varphi
$$$$
\chi_{s}
$$

\section{Subscripts}

$i$

max

mol

$n$

o

$t$

p particle/aggregate surface area concentration, $\mathrm{m}^{2} / \mathrm{m}^{3}$

particle/aggregate surface area, $\mathrm{m}^{2}$

particle/aggregate projected surface area, $\mathrm{m}^{2}$

projected area exponent

mass fractal dimension

mass-mobility exponent

surface fractal dimension

diameter of gyration, $\mathrm{nm}$

mobility diameter, $\mathrm{nm}$

equivalent primary particle diameter, $\mathrm{nm}$

peripheral fractal dimension

soot volume fraction

Boltzmann constant, $\mathrm{m}^{2} \mathrm{~kg} \mathrm{~s}^{-2} \mathrm{~K}^{-1}$

diffusive Knudsen number

projected area prefactor

mass fractal dimension prefactor, $\mathrm{kg} \mathrm{m}^{-\mathrm{Df}}$

mass-mobility prefactor, $\mathrm{kg} \mathrm{m}^{-\mathrm{Dfm}}$

surface reaction constant, $\mathrm{m}^{3} \mathrm{~mol}^{-1} \mathrm{~s}^{-1}$

particle/aggregate mass, $\mathrm{kg}$

mass of two carbon atoms, $\mathrm{kg}$

number concentration, $\mathrm{m}^{-3}$

Avogadro number, mol $^{-1}$

number of particles/aggregates

number of equivalent primary particles per aggregate/agglomerate surface reaction rate, $\mathrm{m}^{-3} \mathrm{~s}^{-1}$

temperature, $\mathrm{K}$

residence time, $\mathrm{s}$

particle/aggregate volume, $\mathrm{m}^{3}$

collision frequency, $\mathrm{m}^{3} \mathrm{~s}^{-1}$

particle-molecule collision efficiency

fraction of active surface sites

soot bulk density, $\mathrm{kg} \mathrm{m}^{-3}$

geometric standard deviation of mobility diameter

equivalence ratio

number density of soot surface radicals, $\mathrm{m}^{-2}$

particle/aggregate indice

maximum

molecule

after reaction of one acetylene molecule

initial

total

primary particle 


\section{Overview}

\subsection{Combustion for Energy Generation \& Material Synthesis}

Traditionally combustion science and engineering is focused on energy generation. So particle or soot formation tends to be viewed as a nuisance. As a result, the corresponding research focuses typically on the interplay of chemical reactions and fluid mechanics that lead to soot formation and growth [1,2]. Very recently emphasis has been placed on soot morphology for reliable monitoring and eventual mitigation, if not, suppression of soot emissions [3, 4].

In contrast, in material synthesis, the goal is the reproducible generation of "soot" or, more accurately, particles with well-defined characteristics: size, crystallinity and morphology. In fact, the term soot is rarely used since such combustion-generated particles have market value. In most of their applications, performance is determined by the primary particle size and crystallinity. Aside from on-line monitoring, in material synthesis soot or particle morphology is both a solids processing and a performance issue, especially when it involves chemically-bonded agglomerates, the socalled aggregates. These are quite attractive in electroceramics (gas sensors, battery materials) as they facilitate electron transport [5] by minimizing contact resistance that is critically important for the performance of the corresponding devices. Even for commodities like $\mathrm{TiO}_{2}$, the proximity of aggregate (or optical) size to primary particle (or rutile crystallite) size determines the value of the product pigment. Furthermore aggregates are quite attractive in the manufacture of catalysts and optical fiber preforms as they facilitate transport of reactive and product gases (in catalyst beds) or undesirable by-product $\mathrm{H}_{2} \mathrm{O}$ and $\mathrm{HCl}$ (in lightguide preforms) to/from their surfaces [5].

Today combustion contributes decisively to the manufacture of commodities such as carbon black, optical fibers, widely-used fumed oxides $\left(\mathrm{SiO}_{2}, \mathrm{Al}_{2} \mathrm{O}_{3}\right.$ etc. $)$ and most of white $\mathrm{TiO}_{2}$ pigments, a $\$ 15 \mathrm{~B}$ industry worldwide, at "soot" production rates of $25 \mathrm{t} / \mathrm{h}$ [6]. Most of this industry, however, was built by evolutionary research in the last century as highlighted by Cabot [7] on the history of carbon black, Ulrich [8] on flame-made oxides, Pratsinis and Mastrangelo [9] on aerosol reactor design, Kühner and Voll [10] on carbon black synthesis and Rosner [11] on various flame-made particles and films. The early development of material synthesis by combustion was difficult. Reactors employed exotic materials (some $\mathrm{TiO}_{2}$ plants were the world's largest inventories of Pt) and frequently had serious setbacks, most notably by particle deposits on reactor walls and eventual clogging [12].

Since the 70's systematic research has been carried on the fundamentals of synthesis [13] and photocatalytic applications of flame-made materials [14]. The latter could have contributed to the manufacture and broad use of the "gold standard" for photocatalysis, the so-called $\mathrm{P} 25 \mathrm{TiO}_{2}$ by Degussa (and now Evonik). It is not clear how fundamental research contributed to growth of the industry of flame-made materials [12]. Brilliant research results [13] were "casually" published by the IP-conscious industry at the time without probably appreciating their value. Most likely, the inability to connect particle dynamics with reactor fluid mechanics through systematic computational methods at the time 
might have prevented a quantitative connection between lab scale and production scale reactor data and, subsequently, a functional understanding of the process from first principles [12].

Nevertheless such research sparked keen interest in industry and academia to better understand combustion aerosols for the efficient manufacture of the above commodities and, most recently, for synthesis of far more sophisticated material compositions for a wide spectrum of applications [5]. Several technical and tutorial reviews have been published on the fundamentals of flame aerosol synthesis of materials [15-17], computational methods [18], reactor design [19], spray combustion fundamentals and products [5], flame spray precursors [20], early stage particle formation [3], flame-made catalysts [21-23], breath sensors [24], characterization methods [25] of flame-made particles and the assembly of such films and devices [26].

\subsection{Flame aerosol technology in material synthesis}

Currently combustion synthesis of materials is driven by the rapidly advancing understanding of this technology and, most importantly, by its proven scalability and capacity to stably produce unique (carbon-coated metals for biomagnetic nanofluids [27]) and even metastable materials, e.g. like $\varepsilon-\mathrm{WO}_{3}$ for breath acetone sensing [28] and low temperature $\mathrm{BaCO}_{3}$ for $\mathrm{NO}_{\mathrm{x}}$ removal by nitrogen storage-reduction [29], inaccessible by conventional wet-chemistry and annealing that lead to the corresponding, thermodynamically-dictated phase compositions. Furthermore, combustion does not involve the tedious steps of wet chemistry and can produce high purity materials (e.g. optical fibers) without environmentally burdensome liquid by-products [9]. On the other hand, for certain material compositions, suitable precursors for solid particle synthesis are relatively expensive while highly crystalline and porous materials are difficult to produce [23]. As a result, an array of new flame-made materials and applications [5] are discovered that are brought to market by start-ups addressing niche markets like carbon nanotubes (Nano-C) [30], nanosilver (HeiQ) [31] and biomagnetic nanofluids (TurboBeads) [32] to name a few. For example, $500 \mathrm{mg}$ of flame-made carbon-coated cobalt [27] that is amine-functionalized by a subsequent wet process is sold at $\$ 137$ by Sigma-Aldrich (product \#742406) translating to $\$ 274^{\prime} 000 / \mathrm{kg}$ while its $\mathrm{kg}$ quantities are available at $\$ 50 ’ 000 / \mathrm{kg}$ when gold sells for just over $\$ 42^{\prime} 000 / \mathrm{kg}$. Further functionalization can bring the value of such magnetic nanoparticles to $\$ 2^{\prime} 800^{\prime} 000 / \mathrm{kg}$ (TurboBeads PEG-Amine \#4004) again at small product quantities [33]. In fact, the scalability of flame spray technology to $\mathrm{kg} / \mathrm{h}$ has been demonstrated in academic laboratories [34] which has encouraged mainstream catalyst manufacturers like JohnsonMatthey to build those in house [35] along with diverse communities and state laboratories worldwide [36].

So it is not difficult to imagine that there is a wealth of new flame-made products in the market and even more in the "pipeline" so-to-speak. Today a score of nanomaterials (films and particles) can be readily made in flames with closely controlled size, composition and morphology for catalysis, gas sensing, phosphors, battery and fuel cell 
electrodes, dental and phosphetic biomaterials and even nutritional supplements [5]. This is driven now by a quantitative understanding of flame spray synthesis of nanoparticles [37] from the individual droplet mixture evaporation or explosion [38] to actual reactor units manufacturing them at $\mathrm{kg} / \mathrm{h} \mathrm{[39].} \mathrm{In} \mathrm{fact,} \mathrm{various} \mathrm{flame-made} \mathrm{particle} \mathrm{compositions}$ and sizes are readily available on the internet that customers can easily select using the periodic table and place an order through user-friendly webpages (nanograde) [40].

\subsection{Key advances in aerosol science enabling material synthesis}

In the last 20-30 years there have been distinct advances in both the understanding and creation of unique materials by combustion. For example, combustion deposition of extremely pure and closely-controlled glass composition by the vapor axial deposition and outside vapor deposition processes enabled the manufacture of lightguides (with well-defined refractive index) that did not require frequent signal amplification creating the optical fiber industry [41]. By evaporation and combustion of metal halide mixtures, the light absorbing impurities (e.g. Fe) stayed practically in solution resulting in pristine silica particles for light transmission. This led to optical fibers being a commodity today, in contrast to the hi-tech material in the early 80 's. The use of multiple laminar flow aerosol reactors during lightguide preform fabrication by modified chemical vapor deposition [42], allowed, for the first time, the use of combined fluid and aerosol particle dynamics [43] in the process design for the manufacture of optical fibers.

A second advance came with the understanding that the formation of filamentary refractory particles was due to the interplay of coagulation and sintering [44]. So by closely controlling the high temperature particle residence time (HTPRT), it was possible to control the morphology of flame-made particles, from fractal-like to compact, nonaggregated structures [16]. For example, by decreasing the HTPRT in vapor-fed diffusion flames through increasing their oxidant flow rate, silica particles became increasingly fractal [45], while $\mathrm{TiO}_{2}$ behaved similarly by reducing the oxygen content of the oxidant stream at constant flow rate [46].

A third advance was the realization that during material synthesis in flames by gas-to-particle conversion, newly-formed particles grow rapidly by coagulation and attain an asymptotic or self-preserving size distribution (SPSD) that does not change even though particles continuously grow. This allowed simple models of particle dynamics [47] to be interfaced with fluid mechanics for quantitative understanding of the role of process variables (e.g. reactant concentration and mixing pattern [48]) on product $\mathrm{TiO}_{2}$ [49]. Such models follow the dynamics of a single moment of the distribution (e.g. number) and by a mass balance (as the aerosol mass is conserved, e.g. no soot oxidation to $\mathrm{CO}_{2}$ ) the average particle diameter is obtained. By scaling the SPSD to that average particle diameter and number concentration, the full size distribution is obtained. That way the dramatic impact of reactant mixing on changing fractal-like anatase $\mathrm{TiO}_{2}$ particles to 10 times larger non-fractal rutile ones [48] at the same reactor and gas flow rates could be explained 
theoretically [49] and confirmed experimentally by small angle x-ray spectroscopy [50]. It should be noted that process design here is not that of classic combustion modeling where reaction rates are determined rigorously for determination, say, of flame temperature from first principles. Modeling flame synthesis of materials typically offers a template relating process variables to product characteristics [19]. In fact, manufacture of even "simple" products like pigmentary $\mathrm{TiO}_{2}$ involves many additional components like traces of $\mathrm{Al}$ to assure formation of the rutile as well as trace metals $(\mathrm{Na}, \mathrm{K}$ etc.) to control its state of aggregation [51] or minimize deposition to reactor walls [12]. As a result, material properties (e.g. sintering rate) required in such models are unknown. By model comparison to a data subset, "effective" sintering rates can be obtained [52] and used quite effectively in the process parameter space (temperature, cooling rate and concentration) for selecting product characteristics (primary particle size and extent of aggregation or hardagglomeration) [53].

A fourth advance came with the use of thermophoretic sampling [54] during combustion synthesis of materials. This allowed direct observation of the growth evolution of flame-made particles, without relying on assumptions on size distribution or ex-situ microscopy to invert optical data. That way, the detailed evolution of particle growth in premixed flames is revealed for $\mathrm{TiO}_{2}$ [55] or the so-called secondary nucleation of fumed $\mathrm{SiO}_{2}$ is traced to mixing of particles generated in different streamlines [56]. Also chemical pathways for particle formation are determined as well as the error associated with the use of the above simple models in comparison to detailed population balances [55]. So once particle formation has been completed, the error in using a monodisperse rather than a detailed model for particle dynamics is a mere $20 \%$ [57] which is typically within the accuracy of aerosol instruments.

A fifth advance came with the systematic development of flame spray pyrolysis (FSP), or more accurately, spray combustion [58]. This made possible the synthesis of materials with select compositions and applications [5] from an array of precursors [20]. Spray combustion is the workhorse for manufacture of carbon black, by the so-called furnace process since the 1940s [10]. Various refractory nanoparticles had been made by spray combustion (e.g. $\mathrm{ZnO}$ [59], $\mathrm{Al}_{2} \mathrm{O}_{3}$ [60], $\mathrm{Al}_{2} \mathrm{O}_{3} / \mathrm{SiO}_{2}$ [61], $\mathrm{MgAl}_{2} \mathrm{O}_{4}$ [62]). However, its widespread use for synthesis of a variety of nanoparticles and films, took place after recognizing that gas-to-particle conversion led to synthesis of solid particles with uniform composition [63]. So by capitalizing on the quantitative understanding of gas-to-particle conversion [64], an array of particle compositions were made up to $\mathrm{kg} / \mathrm{h}$ even in academic labs (e.g. $\mathrm{SiO}_{2}$ [34], $\mathrm{ZrO}_{2}, \mathrm{Fe}_{3} \mathrm{PO}_{4}$ [36]) as the availability of gaseous precursors was no longer an obstacle. The FSP process led to novel catalysts [23], electroceramics (e.g. gas sensors) and biomaterials making combustion a bona fide route for scalable synthesis of broad material compositions [5], way beyond the flame-made commodities discussed earlier on. Spay combustion is practiced gainfully by a number of spinoffs and is investigated actively by several academic and industrial laboratories today [36]. For example, starting in mid-2017, the German National Science Foundation plans to support about $30 \mathrm{PhD}$ projects on the fundamentals of FSP 
for six years.

\subsection{The relationship between the science of soot formation and aerosol synthesis of materials}

As stated earlier, a focus of classic combustion science is on possible chemical pathways for soot formation to essentially block them as the release of soot represents lost energy and, most notably, pollution. In principle, there is little interest on soot morphology during hydrocarbon combustion for energy generation besides accurate monitoring and relating soot emissions to combustion process variables. In contrast, material synthesis focuses on the formation of such "soot" and product particle size, composition and morphology that depends largely on the end use of such particles or films. Primary particle or crystal size is important in almost all applications as it determines, for example, the catalyst surface area or the electroceramic performance of a semiconductor or the antibacterial activity of a nanosilver composition. The extent of aggregation is also important. Soft or physically-bonded agglomerates are needed for nanoparticles in liquid suspensions (e.g. slurries or paints) or solid matrixes (nanocomposites) while hard-agglomerates or aggregates (section 2.1) are important for catalysts, lightguides and electroceramic devices.

In both energy generation and material synthesis there is significant interest in understanding the early stages of particle formation and growth. This includes particle and/or crystallite nucleation or inception and the so-called nascent soot growth before the attainment of any asymptotic fractal-like structures or size distributions. For example, in flame synthesis of $\mathrm{TiO}_{2}$ there is strong interest in forming the rutile phase for pigments while anatase is desired for catalysts and phototcatalysts. Typically this is accomplished by controlling the oxidant composition in the flame [46] or by the addition of dopants [51]. For example, $\mathrm{Al}$ or Sn promote rutile and Si promotes anatase through substitutional or interstitial defects, respectively [65]. However the detailed understanding of how such crystal formation takes place during combustion is missing, even though there is significant effort in this direction [66]. This is important, also, in forming binary or ternary oxide supports for heterogeneous catalysts and even selective gas sensors that cannot be easily made by conventional wet chemistry.

The dynamics of the mesmerizing and omnipresent fractal-like structures of nanoparticles are important also in both energy and materials applications of combustion. Today these dynamics are better understood and used to accurately monitor such structures and systematically design and operate their manufacturing units. For example, novel computational schemes capitalizing on mesoscale or Discrete Element Modeling (DEM) reveal that power laws govern the evolution from physically-bonded soft agglomerates of nanoparticles to chemically- or sinter-bonded hardagglomerates or aggregates, regardless of material composition and polydispersity [67]. Understanding is rapidly advancing also at the sub-particle level, molecular or atomistic, with clever algorithms and hardware revealing detailed sintering or coalescence mechanisms [68] and even the dynamics of crystallinity development from first principles [69] 
on par with such efforts in soot dynamics [70]. In material synthesis this facilitates the development of process design correlations rigorously tested with data gradually placing flame aerosol technology on a firm scientific basis [26]. Techniques developed for soot monitoring such as tandem mass-mobility measurements not only quantify the above agglomerate structures but give the size of constituent nanoparticles when interfaced with the above power laws [71]. That way the nanoparticle size, a key performance characteristic in almost all applications, is given online rather than from tedious offline microscopic or adsorption measurements that in many ways hinder efficient manufacturing due to the time-lag from measurement to response.

Last but not least, is the relationship between environmental impact and technological applications of combustion aerosols in actual process operation and product handling and development. This is important for better understanding the health impact of flame-made nanomaterials for both practitioners and clients or users. Today the public is far more cautious with scientific discoveries asking for proof that they are human-friendly before embracing them. For example, just a few years ago the Environmental Protection Agency of United States had been petitioned to label nanosilver (silver nanoparticles) as a pesticide for its impact on aquatic life [72]. Such a material labeling is the "kiss to death" so-to-speak of any consumer product. Flame synthesis of nanosilver with controlled size, however, clearly differentiated the role of silver ions and particles [73], contributing effectively to environmental policy on new nanomaterial development. Today nanosilver is the largest engineered nanomaterial in the market after the classics, carbon black and fumed oxides.

Being aware of possible health implications of certain materials closely guides the corresponding research and development [74]. For example, a number of passive measures are implemented to assure the health of researchers in combustion aerosol laboratories for material synthesis as frequently unknown compositions are generated and handled. So, researchers are required to wear masks and appropriate clothing while labs operate at 20-30 Pa below atmospheric pressure, similar to bio labs, to assure no release of such nanoparticles. Furthermore certain compositions (e.g. Ni and Cr) are either avoided altogether or handled by enclosing flame reactors in airtight gloveboxes while chemical storage of reactants is continuously vented and closely monitored.

\subsection{Devices}

The capacity of flame aerosol technology for inexpensive synthesis of functional nanoparticles with closely controlled characteristics motivates their incorporation into sophisticated devices [26]. The use of such relatively lowcost nanoparticles in the assembly of nanostructured and portable devices is a frontier of engineering today as most of current industrial applications involve passive nanoparticles in the form of powders or suspensions. Though the assembly of a number of such devices is explored for fuel cells, batteries and nanogenerators, flame-made gas sensors for breath 
analysis have been advanced to industrial prototypes and will be highlighted here.

In general, gas sensors are semiconducting films $\left(\mathrm{SnO}_{2}, \mathrm{TiO}_{2}, \mathrm{WO}_{3}\right.$ etc. $)$ on standard microeletronic circuitry. Upon exposure to gas mixtures at appropriate temperature, the gas analyte of interest is detected selectively by its reaction on the semiconductor surface (similar to heterogeneous catalysts) by monitoring the accompanying change in resistivity through the microeletronic circuit. Key issues in this technology are the sensitivity, selectivity and sensor response and recovery times over prolonged periods [75]. Combustion contributes to this field in three ways. First, by flame synthesis of such $\mathrm{SnO}_{2}$ [76], $\mathrm{ZnO}$ [77] or $\mathrm{TiO}_{2}$ [78] and even direct deposition onto microeletronic substrates with rapid heat removal resulting in crack-free and highly porous sensing films [79]. The flame synthesis and direct deposition of sensing nanoparticles onto sensor substrates greatly simplifies the deposition of sensing films as there is no need for particle suspension in slurries or pastes followed by drying and long annealing for the crystalline phase development required in conventional sensor film synthesis by spray deposition or doctor-blading [5]. Second, by rapid in-situ flame annealing of such films through microelectronic masks [80] to enhance film cohesion and adhesion on the underlying noble metal circuitry and diverse substrates (alumina or silicon). Then the resulting sensing films can withstand the demanding processing (e.g. diamond cutting, jet washing, polishing etc.) of that industry forming transparent $\mathrm{SnO}_{2}$ gas sensors onto complementary metal oxide semiconductor (CMOS) integrated circuitry, containing temperature sensors and Pt-heaters [81]. Both of these ways have shown that flame-made sensors are as good (if not better) as those made conventionally for detection of $\mathrm{CO}, \mathrm{NO}$ and other gases and organic vapors [76-79]. Being, however, as good or slightly better is not enough to displace existing sensor fabrication technologies by flames.

Third and, probably, the most important way that flame technology contributes to gas sensor development comes with its capacity to stably form uniquely metastable phase compositions of sensing films. This makes possible to selectively detect compounds down to ppb in the multicomponent mixture (1'000+ organic vapors and gases) of human breath at high relative humidity (RH) [24]. Such compositions are not readily accessible by conventional wet chemistry processing. So nanostructured and metastable $\varepsilon-\mathrm{WO}_{3}$ films stabilized by Si-doping can sense acetone down to $20 \mathrm{ppb}$ at 90\% RH [28], a tracer for diabetes type-1 and, most recently, for body fat burning. Also such flame-made films of Sidoped $\mathrm{MoO}_{3}$ can detect $\mathrm{NH}_{3}$, a tracer for end stage renal disease, down to $400 \mathrm{ppb}$ [82] and Ti-doped $\mathrm{ZnO}$ that can detect isoprene, a tracer for blood cholesterol, down to $3 \mathrm{ppb}$ [83] at these RH conditions and at high signal-to-noise ratios (SNR). Miniaturizing such sensors into portable devices has facilitated their testing with humans online [84] and offline revealing their potential for reliable detection of breath acetone on par with standard glucose tests [85]. This motivates the development of industrial prototypes [86] for clinical tests that can hopefully phase out the painful finger pricking for glucose testing of diabetics. In a lighter application, such sensors could show also when a gym workout is burning (un)desirable body fat as breath acetone is also a tracer of such metabolism. 
The capacity of flame technology to generate such semiconducting particles of different sizes and compositions motivates also the development of sensor arrays, the so-called electronic nose or E-nose. Such units capitalize on the variability of the response of various sensors to selectively detect compounds in multicomponent mixtures without requiring the unique phase composition and selectivity of the above semiconductors. E-noses are attractive for screening large populations for security and biomedical applications. For example, highly sensitive but non-selective $\mathrm{SnO}_{2} \mathrm{sensors}$ doped with Pd, Pt, Ti and Si in such an E-nose can detect formaldehyde, a tracer for lung cancer and indoor air pollution, down to $5 \mathrm{ppb}$ at high SNR and high RH in mixtures of ethanol, acetone and $\mathrm{NH}_{3}$ having an order of magnitude higher concentrations than formaldehyde [87]. The availability of such sensors, for example, can facilitate routine pre-MRI screening of people for early stage diagnosis of cancer. These developments point out the potential of flame synthesis and direct deposition of nanostructured functional layers on temperature-sensitive supports at ambient pressure that could be attractive for other devices as has been demonstrated already with flexible multi-layered [88] and even electricallyconductive polymer nanocomposite films [89].

\section{Nascent Soot Growth Dynamics}

Nascent soot growth dynamics are important in all flame-made carbon-containing functional nanostructured materials. Examples include $\mathrm{C} / \mathrm{SiO}_{2}[90,91]$ and $\mathrm{C} / \mathrm{TiO}_{2}$ [92] for nanocomposites, $\mathrm{C} / \mathrm{Cu}$ for gas sensors [93], surface-functionalized C/Co [94] and $\mathrm{C} / \mathrm{Fe}_{3} \mathrm{C}$ [95] for biomagnetic nanofluids, C-coated $\mathrm{LiMn}_{2} \mathrm{O}_{4}$ [96] and $\mathrm{LiFePO}_{4}$ [97] for battery electrodes, $\mathrm{Pt} / \mathrm{C}$ [98-100] and Pt-Ru/C [101] catalysts for fuel cells. Furthermore, the size distribution and morphology of nascent soot determine the specific surface area [102] and thus the performance and quality of carbon black, the largest by value and volume nanostructured material made by combustion today [6]. In addition, surface growth and agglomeration is encountered in other flame-made materials like $\mathrm{TiO}_{2}$ [103]. Most importantly, the extent of chemical bonding (e.g. aggregate or hard-agglomerate formation) between nascent soot primary particles determines their conductivity and thus device performance such as fuel cells [104] and gas sensors [105]. Thus, good understanding of nascent soot formation by agglomeration and surface growth through Discrete Element Modeling (DEM) could facilitate the design of such flame-made functional nanostructured materials. In fact, the performance of such models can be tested with classic soot formation experiments, where detailed characterization of soot size, morphology and concentration routinely takes place [4]. So, flame synthesis of materials having a clear focus on particles with closely controlled characteristics can contribute to advancing the understanding of nascent soot formation by agglomeration and surface growth through mesoscale or DEM simulations as superior databases are created in soot formation studies for energy generation $[3,4]$ (section 2.2). 
According to Dobbins [106], soot can be classified in two categories based on its formation stage, namely nascent (particles of 1-10 nm mobility diameter) and mature soot (fractal-like aggregates with mobility diameters between 10 and $100 \mathrm{~nm}$ ). Nascent soot particles have been observed in a wide range of combustion sources, from laminar diffusion [107] and premixed flames [108] to diesel engines [109]. There are major concerns about the adverse effects of nascent soot particles on public health due to their small size and high specific surface area [110]. For example, the fraction of soot particles that deposit in the human lung is increasing for decreasing mobility sizes [111]. Furthermore, the reactivity of nascent soot particles correlates with their high surface area, inducing the presence of toxic species on their surface [110]. Thus, a better understanding of nascent soot formation is required for identification of process parameters that determine soot particle size and morphology.

Various theories describe the formation of nascent soot $[112,113]$. Typically, small soot particles are considered to be in a liquid-like state, coalescing rapidly into a single sphere upon collision [112]. These particles are solidified by carbonization (loss of hydrogen) and coagulate into fractal-like structures [112]. Reilly et al. [114] identified primary particles of mature soot smaller than spherical nascent soot collected from the same flame. Furthermore, the polydispersity of nascent soot mobility size depends strongly on flame conditions [115]. However, the width of mature soot primary particle size distribution obtained by microscopy was not sensitive to the equivalence ratio of laminar premixed flames [116].

Mitchell and Frenklach [113] suggested that spherical nascent soot particles are the product of agglomeration and surface growth taking place after nucleation. Schenk et al. [117] supported this by microscopic measurements and showed that nascent soot has, in fact, irregular morphology in a wide range of sizes (7-30 nm). Mass-mobility measurements by Camacho et al. [4] further revealed that nascent soot has up to three times less mass than a compact sphere of the same mobility size, consistent with sizing of fractal-like particles [118]. This relationship between mass and mobility cannot be interpreted by a constant geometric shape, including well-defined fractal structures and straight chains of primary particles [4]. This indicates the need of a more detailed accounting of the evolution of nascent soot structure during simultaneous surface growth and agglomeration or aggregation.

Many numerical studies of the evolution of nascent soot size distribution neglect its morphological changes up to $20-35 \mathrm{~nm}$, assuming either spherical $[119,120]$ or asymptotic (constant) fractal-like structures similar to mature soot [121,122]. Monte Carlo simulations based on the theory of Mitchell and Frenklach [113] neglecting cluster-cluster agglomeration, provided a relationship for the radius of gyration as function of the surface fractal dimension describing the transition from spherical to fractal-like structures [123]. Morgan et al. [124] extended the model of Mitchell and Frenklach [113] to account for collisions between agglomerates and traced the temporal evolution of soot aggregate volume-equivalent and gyration radii, primary particle diameter, mass and surface fractal dimension in laminar premixed 
flames. However, their size distributions were broader than the corresponding experimental data due to increased coagulation and surface growth rates [124].

Here, nascent soot growth is investigated by DEM of agglomeration and surface growth by acetylene pyrolysis. The model is validated with theoretical expressions for agglomeration alone [125] as well as with surface growth with and without coagulation at full coalescence. The evolution of nascent soot structure is benchmarked against previous numerical studies $[123,124]$. Soot growth by agglomeration with or without surface growth is compared to coagulation at full coalescence in terms of mean mobility diameter, polydispersity and morphology. The evolution of mobility size distribution and morphology of soot growing by agglomeration and acetylene surface growth are compared to microscopy [117] and mass-mobility measurements [4] of a benchmark burner-stabilized stagnation ethylene flame. Then, the effect of soot volume fraction on nascent soot morphology is elucidated and, most importantly, a scaling law is proposed for determining the nascent soot primary particle size from mass-mobility measurements.

\section{Theory}

\subsection{Reaction Model}

Acetylene is the major gaseous species produced by pyrolysis of ethylene in the first few millimeters of laminar flames $[3,126,127]$. Acetylene molecules contribute to soot growth by the Hydrogen Abstraction-Carbon Addition (HACA) mechanism [128]. Their reaction rate, $r_{i}$, with soot aggregate $i$ of surface area $a_{i}$ and number density $N_{i}\left(\# / \mathrm{m}^{3}\right)$ is [129]: $r_{i}=\frac{k_{s} \chi_{s} N_{m o l} \theta}{N_{a v}} N_{i} a_{i}$

where $N_{m o l}\left(\# / \mathrm{m}^{3}\right)$ is the acetylene concentration, $N_{a v}(1 / \mathrm{mol})$ the Avogadro number, $\chi_{s}\left(\# / \mathrm{m}^{2}\right)$ the number of surface radical sites on one soot particle or aggregate (or agglomerate) per unit surface area produced by surface reaction with hydrogen atoms [129], $\theta$ the fraction of active sites and $k_{s}\left(\mathrm{~m}^{3} /(\mathrm{mol} \cdot \mathrm{s})\right)$ the surface reaction rate constant for acetylene addition [127]:

$k_{s}=80 \cdot T^{1.56} \exp \left(-\frac{1912.4}{T}\right)$

where $T(\mathrm{~K})$ is the temperature.

Here, condensation of Poly-Aromatic Hydrocarbons (PAH) on the soot surface is neglected due to their relatively low concentration compared to acetylene $[121,130]$ and soot oxidation is neglected as only relatively short residence times (or low heights above the burner, HAB) are considered. Also soot aggregates consist of chemically-bonded, spherical-like primary particles, while agglomerates consist of physically-bonded ones and/or aggregates. 


\subsection{Surface Growth and Polydispersity}

When acetylene collides with, say, spherical soot particles, their collision rate, $\beta_{m o l}$, follows the kinetic theory of gases [131]:

$\beta_{m o l}=\pi\left(d_{p}+d_{m o l}\right)^{2} \sqrt{\frac{k_{B} T}{2 \pi}\left(\frac{1}{m_{p}}+\frac{1}{m_{m o l}}\right)}$

where $d_{p}$ and $d_{m o l}$ are the diameters of the soot particle and the acetylene molecule, respectively, and $m_{p}$ and $m_{m o l}$ their masses. The particle growth rate is [131]:

$\frac{d m_{p}}{d t}=\gamma \beta_{m o l} N_{m o l} m_{m o l} \leftrightarrow \frac{d v_{p}}{d t}=\frac{\gamma \beta_{m o l} N_{m o l} m_{m o l}}{\rho_{s}}$

where $v_{p}$ and $\rho_{s}$ are the soot particle volume and bulk density, respectively, and $\gamma=r_{i} /\left(\beta_{m o l} N_{i} N_{m o l}\right)$ the fraction of effective collisions determined by the ratio of the reaction kinetics over the particle-molecule collision dynamics. Since $\gamma$ is proportional to $r_{i}$ and thus to $\theta$ (Eq. (1)), which is also a decreasing function of particle size [127], $\gamma$ is also such a function of size. Combining Eqs. (3) \& (4) and writing them in terms of $d_{p}$, the soot particle growth rate in the free molecule regime is:

$\frac{d d_{p}}{d t}=2 \frac{\gamma N_{m o l} m_{m o l}}{\rho_{s}} \frac{\left(d_{p}+d_{m o l}\right)^{2}}{d_{p}^{2}} \sqrt{\frac{k_{B} T}{2 \pi}\left(\frac{1}{\rho_{s} \frac{\pi}{6} d_{p}^{3}}+\frac{1}{m_{m o l}}\right)}$

\subsection{Soot Aggregate or Agglomerate Characterization by Scaling Laws}

The soot mass, $m$, is related to the soot particle diameter of gyration, $d_{g}$, by [132]:

$m=k_{f} d_{g}^{D_{f}}$

where $D_{f}$ is the mass fractal dimension and $k_{f}$ is a scaling prefactor. Similarly, the $m$ scales with the soot particle mobility diameter, $d_{m}$, by [133]:

$m=k_{f m} d_{m}^{D_{f m}}$

where $D_{f m}$ is the mass-mobility exponent and $k_{f m}$ is a scaling prefactor. In the free molecular regime, $d_{m}$ is related to the agglomerate projected area, $a_{a}$, as [134]:

$d_{m}=2\left(\frac{a_{a}}{\pi}\right)^{1 / 2}$

In the free molecular regime, large agglomerates made by Ballistic Cluster-Cluster Agglomeration have $D_{f}=1.91 \pm 0.03$ [135] and $D_{f m}=2.17 \pm 0.10$ [136]. The aggregate surface area, $a$, is related to the aggregate volume, $v$, by [64]: 


$$
\frac{a}{a_{p, o}}=\left(\frac{v}{v_{p, o}}\right)^{D_{s} / 3}
$$

where $a_{p, o}$ and $v_{p, o}$ are the initial primary particle surface area and volume, respectively, and $D_{s}$ is the surface fractal dimension. The smooth surface of a sphere has $D_{s}=2$, while rougher agglomerate surfaces consisting of primary particles in point contact have $D_{s}=3$ [137]. The number of equivalent primary particles per aggregate/agglomerate, $n_{p}$, is related to $a_{a}$ by [138]:

$n_{p}=k_{a}\left(\frac{a_{a}}{a_{a p}}\right)^{D_{a}}$

where $a_{a p}$ is the projected area of a primary particle with diameter $d_{p}$ and $D_{a} \& k_{a}$ are the projected area exponent and prefactor, respectively. Large agglomerates of primary particles in point contact have $D_{a}=1.08 \pm 0.002$ and $k_{a}=1.1 \pm$ 0.002, while aggregates made by sintering have $D_{a}=1.07 \pm 0.03$ and $k_{a}=1 \pm 0.04$ [71] .

\subsection{Numerical Implementation}

Nascent soot dynamics by agglomeration and surface growth are described following the soot Brownian trajectories and neglecting the effect of rotation, van der Waals, electric or hydrodynamic forces [139] by a DEM. So one thousand monodisperse soot particles with initial particle diameter, $d_{p, o}=2 \mathrm{~nm}$, and number density, $N_{t, o}=4.5 \cdot 10^{16} \# / \mathrm{m}^{3}(\mathrm{e} \cdot \mathrm{g}$. nascent soot inception [4]), are randomly distributed in a cubic cell at 1 atm and $1830 \mathrm{~K}$ applying periodic boundary conditions [139]. The initial soot nuclei diameter is the smallest size identified in the inception peak of laminar premixed ethylene flames [4, 117], a typical nuclei diameter in modelling soot dynamics [115] and rather insensitive to the employed equivalence ratios [140] for all soot volume fractions here. The number density of the soot surface radicals, $\chi_{s}$, in each soot particle is obtained using a steady state description for the radicals on the soot surface [3, 129] with constant molar fractions of $\mathrm{C}_{2} \mathrm{H}_{2}$ and $\mathrm{H}_{2}$ of 0.02 and 0.1 , respectively, based on the detailed kinetic model of Saggese et al. [121:

Fig. 6]. The initial number of hydrogen atoms that generate the surface radicals for acetylene surface growth is calculated from the soot mass balance for maximum soot volume fraction, $f_{v, \max }=10^{-8}[108]$. These conditions correspond to the benchmark burner-stabilized stagnation ethylene flame of equivalence ratio, $\varphi=2.07$, studied by Abid et al. [108: Flame C3], Schenk et al. [117] and Camacho et al. [4].

During coagulation, every collision between particles is successful and leads to formation of either a sphere (full coalescence) or a cluster (agglomeration). Particle growth takes place in the free molecular regime, since the gas mean free path is $531 \mathrm{~nm}$ at $1830 \mathrm{~K}$. Furthermore, for the flame conditions studied here acetylene molecules collide with the surface of (primary) particles with initial $d_{p, o}=2 \mathrm{~nm}$ at a rate of $11.610^{22} \# /\left(\mathrm{m}^{3} \mathrm{~s}\right)$ [131], but react only at a rate of 7.6 $10^{19} \# /\left(\mathrm{m}^{3} \mathrm{~s}\right)($ Eq. (1)). Even though the acetylene reaction rate increases as the soot (primary) particles grow, it remains 
slower compared to the acetylene molecule transport, consistent with the experiments of Sunderland and Faeth [141] for low pressure laminar diffusion flames. Thus, the acetylene concentration near the soot particle or aggregate $i$ is practically the same as in the bulk and the conversion rate is described by Eq. (4) [131].

Acetylene reacting with single particles or primary particles of agglomerates/aggregates increases their volume and thus the total soot volume fraction, $f_{v}$. During collisions between agglomerates/aggregates and molecules, surface growth leads to chemical bonding between primary particles (aggregation). After a collision with an acetylene molecule, the new (primary) particle size, $d_{p, n}$, is given by mass balance:

$\pi \frac{d_{p, n}^{3}}{6} \rho_{s}=\pi \frac{d_{p, o}^{3}}{6} \rho_{s}+m_{2 c}$

where $\rho_{s}=1800 \mathrm{~kg} / \mathrm{m}^{3}$ is the soot bulk density, $d_{p, o}$ is the primary particle size before reaction and $m_{2 c}$ is the mass of two carbon atoms contributed by pyrolysis of one acetylene molecule [127]. The resulting soot particle or aggregate velocity is obtained by the respective momentum balance. The DEM method does not assign preferential surface growth near the aggregate neck, since the nascent soot surface is almost uniformly accessible by small acetylene molecules, as shown by Molecular Dynamics [70].

The DEM simulations are compared to those of a moving sectional model (MSM) for coagulation and surface growth [142, 143] using the Fuchs collision frequency [144] for coagulation and Eqs. (1) and (2) for surface growth with $v_{i+1} / v_{i}=1.7$ [143], where $v_{i}$ is the average particle volume of the $i$ th section.

\section{Results and Discussion}

\subsection{Validation}

The MSM is validated with analytical solutions for full coalescence and condensation in the continuum regime [145]. Figure 1a shows the evolution of geometric standard deviation, $\sigma_{g}$, of soot particles having initially lognormal size distributions (SDs) with $\sigma_{g, o}=1.15$ (dotted line and triangles), 1.3 (broken line and squares) and 1.45 (solid line and circles) growing solely by surface growth (SG) as simulated by DEM (lines) and MSM (symbols) simulations. The evolution of $\sigma_{g}$ obtained by DEM closely follows that by the MSM validating the present DEM. Surface growth narrows the DEM- and MSM-derived particle size distributions (SDs) and at sufficiently long times $(t>0.1 \mathrm{~ms})$ they become quite monodisperse $\left(\sigma_{g}<1.05\right)$ for all $\sigma_{g, o}$, consistent with theory $[131,146]$. Thus, both simulations are not affected by numerical diffusion, which is a common problem for simulations of condensation or SG [147].

Figure $1 \mathrm{~b}$ shows the distribution of growth rate, $d d_{p} / d t$, over all particles sizes, $d_{p}$, according to Eq. (5). The growth rate decreases with increasing particle size, showing that small particles grow faster than larger ones narrowing the soot size distribution early on. The collision frequency between soot particles and acetylene actually is higher for 
increasing particle size (Eq. (3)). Bigger particles have larger surface area than smaller ones to undergo more collisions with acetylene. However, the enlargement of particle diameter by these collisions is stronger for smaller than bigger particles, as shown in Eq. (5). Thus, the small particles grow faster than larger ones narrowing the soot size distribution during surface growth.
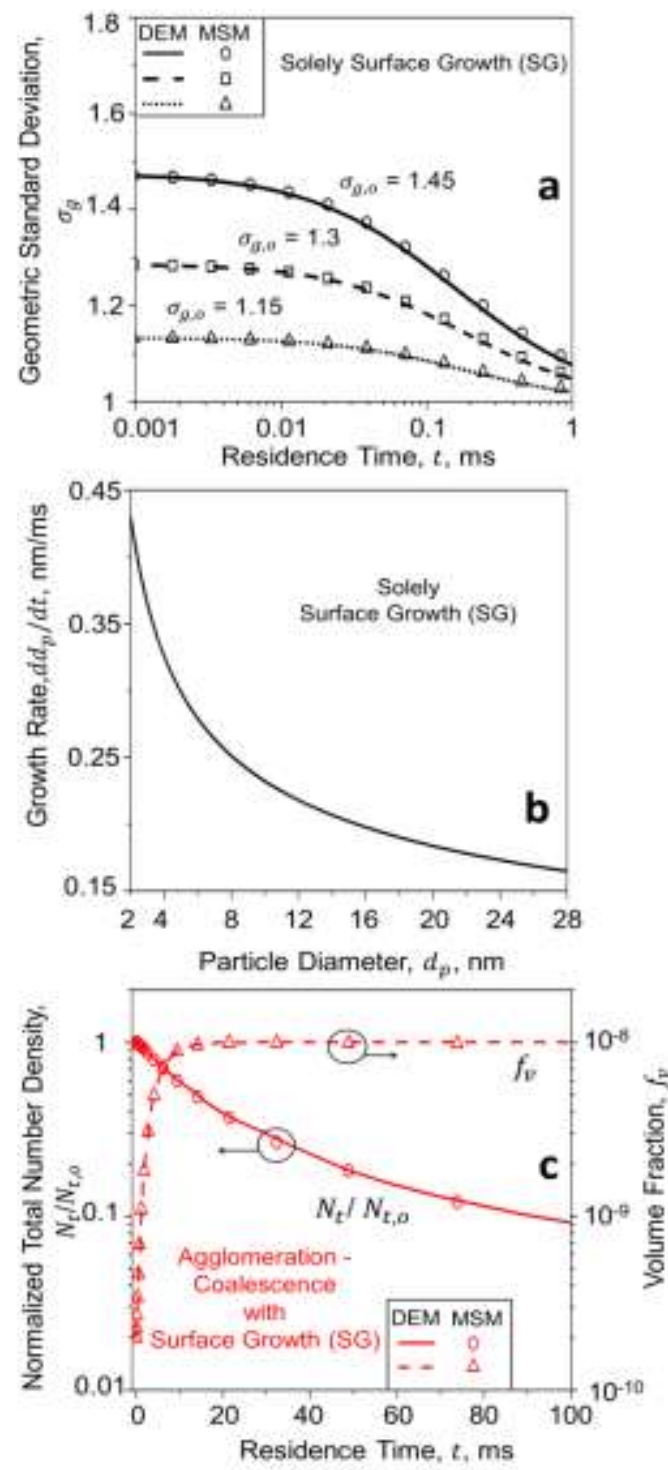

Figure 1. (a) Evolution of geometric standard deviation, $\sigma_{g}$, of soot particles with initial $\sigma_{g, o}=1.15$ (dotted line and triangles), 1.3 (broken line and squares) and 1.45 (solid line and circles) growing by surface growth alone (SG) obtained by Discrete Element Model (DEM: lines) and Moving Sectional Model (MSM: symbols) simulations. At sufficiently long times $(t>0.1 \mathrm{~ms})$ the DEM- and MSM-derived particle size distributions become almost monodisperse $\left(\sigma_{g}<1.05\right)$ for all $\sigma_{g, o}$. (b) Particle growth rate, $d d_{p} / d t$, by surface growth alone in terms of particle size, $d_{p}(\mathrm{Eq}$. (5)). (c) Evolution of DEM- (lines) and MSM-obtained (symbols) normalized total number density, $N_{t} / N_{t, o}$ (solid line and circles), and volume fraction, $f_{v}$ (right-hand axis, broken line and squares), of soot particles growing by full coalescence and SG. DEM is in excellent agreement with the MSM simulations.

Figure 1c shows the evolution of normalized total number density, $N_{t} / N_{t, o}$ (solid line and circles), and volume fraction, $f_{v}$ (broken line and squares, right-hand axis), of spherical soot particles that coagulate at full coalescence and SG obtained by DEM (lines) and MSM (symbols). The soot volume fraction by both models increases reaching its set 
maximum of $f_{v, \max }=10^{-8}$ at $t=10 \mathrm{~ms}$, consistent with Scanning Mobility Particle Sizer (SMPS) experiments [108]. The DEM simulations are in excellent agreement with the MSM-derived $N_{t} / N_{t, o}$ and $f_{v}$ evolution.

Figure 2 shows the evolution of DEM-derived (line) mean diameter of gyration, $\bar{d}_{g}$, as a function of surface fractal dimension, $D_{s}$, of soot particles with initial $d_{p, o}=2 \mathrm{~nm}$ growing by agglomeration and SG. It should be noted that $D_{s}=2$ corresponds to a sphere and $D_{s}=3$ to agglomerates consisting of primary particles in point contact (insets). The soot aggregate roughness represented by $D_{s}$ is increasing by agglomeration and decreasing by surface growth [113]. For $D_{s}<2.15$, soot aggregates grow mainly by SG and collisions mostly between single particles, increasing $\bar{d}_{g}$ only up to $10 \mathrm{~nm}$. In this region DEM is in good agreement with Mitchell [123; squares] indicating the dominance of SG and particle-cluster agglomeration over cluster-cluster agglomeration. For larger $D_{s}$, agglomeration between soot clusters dominates and $\bar{d}_{g}$ increases rapidly up to $28 \mathrm{~nm}$. Mitchell [123] neglected cluster-cluster agglomeration leading to underestimation of $\bar{d}_{g}$, up to $42.2 \%$ at $D_{s}=2.33$ compared to DEM. Morgan et al. [124; triangles] used a numerical simplification for the coagulation kernel that was optimized for spheres and not for the fractal-like structure of soot. Thus, the collision frequency between soot aggregates and agglomerates was overestimated, as it has been stated explicitly [124; triangles], resulting in much larger $\bar{d}_{g}$ than that obtained by DEM or Mitchell [123].

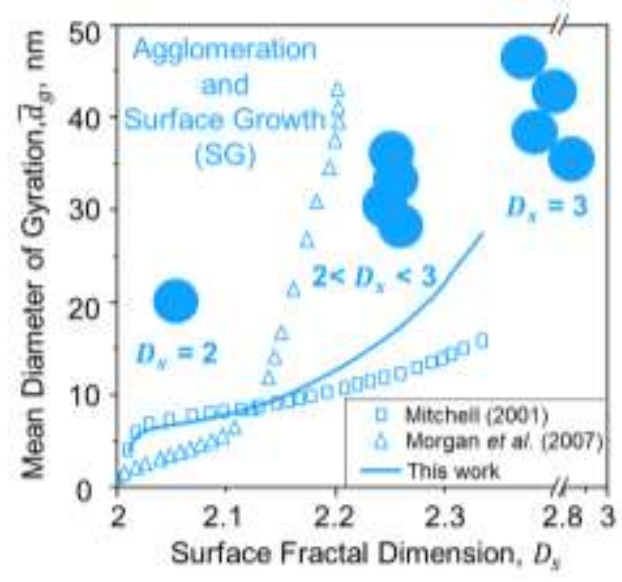

Figure 2. Evolution of DEM-derived (line) mean diameter of gyration, $\bar{d}_{g}$, as function of surface fractal dimension, $D_{s}$, of soot particles growing by agglomeration and surface growth (SG). The results are in good agreement with Mitchell [123; squares] up to $D_{s} \approx 2.15$, while for larger $D_{s}$, the $\bar{d}_{g}$ is underestimated by Mitchell [123] who neglects clustercluster agglomeration. The overestimated agglomeration rate of Morgan et al. [124; triangles] lead to much larger $\bar{d}_{g}$ than those obtained by the present DEM and Mitchell [123]. 
The present DEM simulations are further validated by tracking the collision frequency function, $\beta_{p}$, of soot particles at full coalescence. The DEM-derived $\beta_{p}$ follows closely the Fuchs interpolation for coagulation of monodisperse spheres (not shown here), consistent with Goudeli et al. [125].

\subsection{Effect of Surface Growth on structure, size and polydispersity}

Figure 3 shows snapshots of an exemplary soot particle with $d_{p, o}=2 \mathrm{~nm}$ growing by agglomeration (coagulation) in the free molecular regime (a) without SG, (b) with SG and (c) with full coalescence $\left(D_{f m}=3\right)$ and SG at $t=10,20$ and 30 ms. In the absence of SG (Fig. 3a), all acetylene molecules have been consumed during the inception of soot particles with $d_{p}=2 \mathrm{~nm}$ and $f_{v}=10^{-8}$. These conditions quickly result in fractal-like structures $(10 \mathrm{~ms})$ having many primary particles (e.g. $\bar{n}_{p}=250$ ) and attaining their asymptotic $D_{f m}=2.17 \pm 0.1$ [136]. At $t=30 \mathrm{~ms}$ they reach a mean mobility diameter of $\bar{d}_{m}=50.3 \mathrm{~nm}$ and average number of primary particles per agglomerate, $\bar{n}_{p}=1259$.

When SG takes place (Fig. 3b), compact soot dimers $\left(D_{f m}=2.6, \bar{n}_{p}=1.45\right)$ are formed early on $(t=10 \mathrm{~ms})$ as SG leads to new soot formation on its surface and increases the neck size between primary particles and subsequently the average primary particle size, $\bar{d}_{p}$, from 2 to $7 \mathrm{~nm}$. At $t=20 \mathrm{~ms}$, an aggregate with equivalent $D_{f m}=2.51$ and $\bar{n}_{p}=2.2$ (even though 3 nanoparticles are distinguished) is formed with $\bar{d}_{m}=9.8 \mathrm{~nm}$, similar to the sphere growing by full coalescence and SG (Fig. 3c). At longer residence times when SG has been completed $(t \approx 30 \mathrm{~ms}$, Fig. 3b), agglomeration dominates starting to decrease $D_{f m}$ to 2.49 and increase $\bar{n}_{p}$ to 3.3 , as expected by particle- and clustercluster agglomeration. The so-formed aggregate has larger $\bar{d}_{m}(11.6 \mathrm{~nm})$ than the corresponding sphere $(10.5 \mathrm{~nm}$, Fig. 3c). The necks induced by SG do not grow uniformly between primary particles for increasing $\bar{n}_{p}$ and thus the primary particle size polydispersity in these aggregates increases, in contrast to sintering or coalescence-driven aggregation where the polydispersity of constituent primary particles decreases [67]. At all $t$, the aggregate (Fig. 3b) and the sphere (Fig. 3c) made in the presence of SG have much smaller $\bar{d}_{m}$ and more compact structures (larger $D_{f m}$ ) than particles made solely by agglomeration (Fig. 3a). This is consistent with Kolb and Jullien [148] who showed that reaction-limited mechanisms such as SG lead to smaller and more compact structures than cluster-cluster agglomeration. 


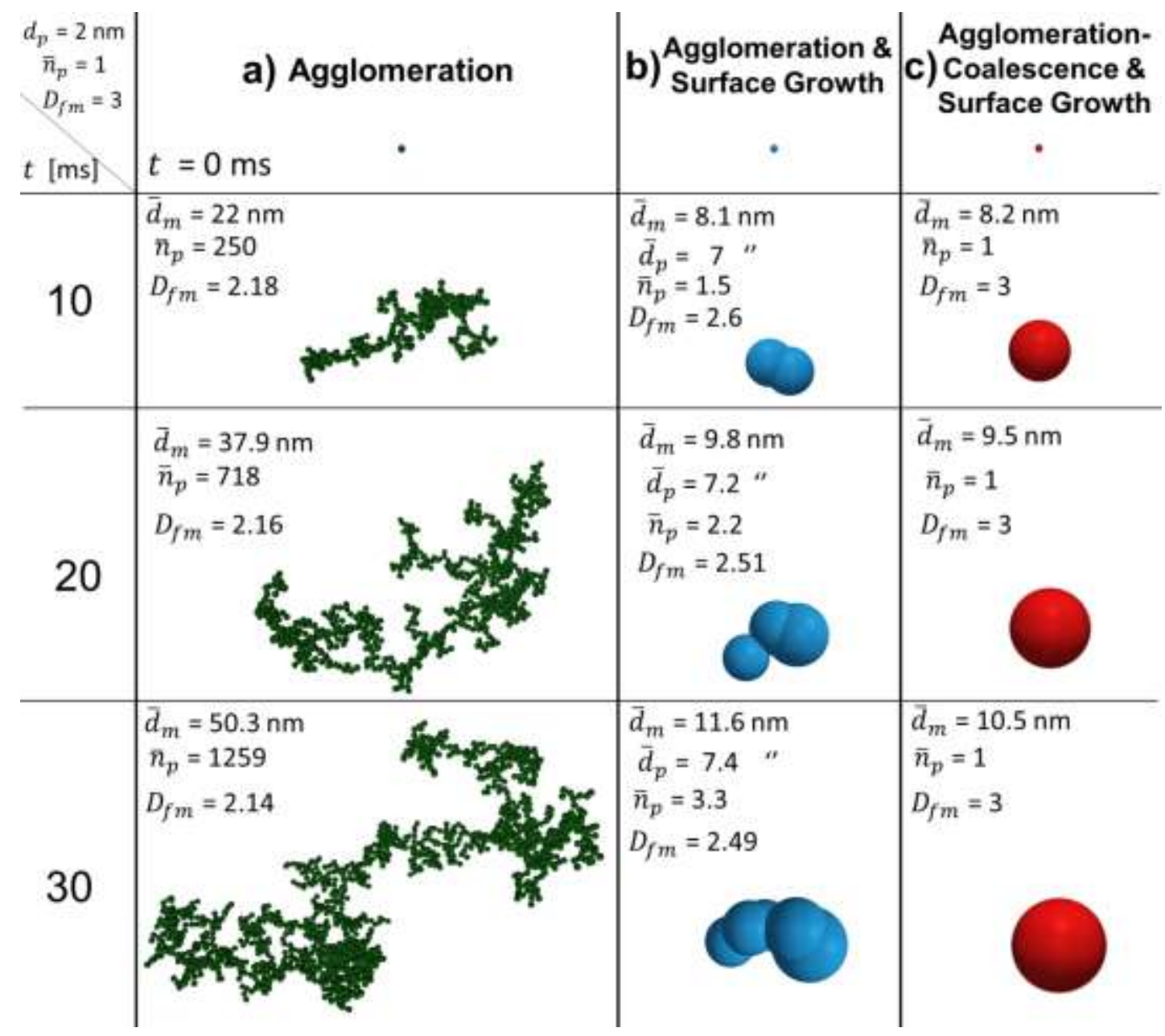

Figure 3. Snapshots of soot particles growing by agglomeration (a) in the absence or (b) in the presence of SG and (c) full coalescence. Particles growing solely by agglomeration increase their average mobility diameter, $\bar{d}_{m}$, from 2 to 50.3 $\mathrm{nm}$ and their average number of primary particles, $\bar{n}_{p}$, from 1 to 1259 , reaching quickly the asymptotic limit of massmobility exponent dimension, $D_{f m}=2.17 \pm 0.1$ [136] by ballistic cluster-cluster agglomeration. When acetylene surface growth takes place on the soot particles (b), these aggregates retain initially their compact morphology $\left(D_{f m}=2.6\right)$, as $\mathrm{SG}$ smoothens the surface of small dimers and increases the average primary particle size, $\bar{d}_{p}$, to $7 \mathrm{~nm}$. For $t<20 \mathrm{~ms}$, agglomeration and full coalescence in the presence of SG (Fig. 3c) produce particles with similar $\bar{d}_{m}$ indicating that SG dominates. At longer $t(t>20 \mathrm{~ms})$, agglomeration progressively dominates decreasing $D_{f m}$ to 2.49 and increasing $\bar{n}_{p}$ to 3.3, while the formed aggregates have larger $\bar{d}_{m}$ than the corresponding spheres. For all $t$, aggregates and spheres made in the presence of SG have much smaller $\bar{d}_{m}$ and larger $D_{f m}(\mathrm{~b}, \mathrm{c})$ than soot particles made solely by agglomeration.

Figure 4 shows the DEM-derived mobility SDs of soot growing by agglomeration (a) in the absence or (b) presence of SG and (c) with full coalescence and SG at $t=10$ (dotted lines), 20 (dot-broken lines) and $30 \mathrm{~ms}$ (solid lines). In the absence of SG and coalescence (Fig. 4a), agglomeration consumes fast the newly-incepted soot monomers with $d_{p}=2 \mathrm{~nm}$ yielding a broad unimodal SD at $t=10 \mathrm{~ms}$ with mobility-based geometric standard deviation, $\sigma_{g, m}=2.01$.

This is approaching the self-preserving size distribution (SPSD) limit of 2.03 for agglomerates coagulating in the freemolecular regime [125]. At $t=20$ and $30 \mathrm{~ms}$, agglomerates coagulate and grow, reaching the transition regime. Their SD 


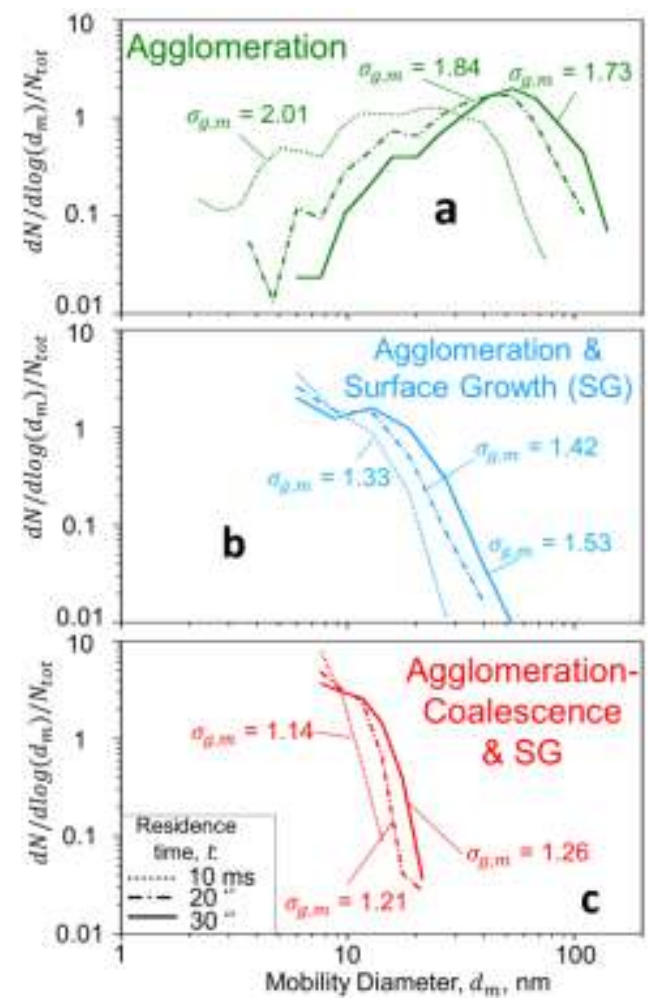

Figure 4. DEM-obtained mobility size distributions (SDs) of soot growing by agglomeration (a) in the absence or (b) in the presence of SG and (c) full coalescence at $t=10$ (dotted lines), 20 (dot-broken lines) and (c) $30 \mathrm{~ms}$ (solid lines). In the absence of SG and coalescence, the formed soot agglomerates attain a broad SD with mobility-based geometric standard deviation, $\sigma_{g, m}=2.01$, close to the self-preserving limit for agglomerates coagulating in the free-molecular regime [125]. Coagulation of large agglomerates in the transition regime narrows the SD up to $\sigma_{g, m}=1.73$ at $t=30 \mathrm{~ms}$. When SG takes place, the soot primary particle size increases from 2 to $6 \mathrm{~nm}$. Agglomeration widens the SD with $\sigma_{g, m}$ up to 1.53 at $t=30 \mathrm{~ms}$. Coalescence reduces further the nascent soot polydispersity, yielding SDs narrower than the selfpreserving limit of 1.46 for spheres in the free molecular regime [150]. Particles growing by agglomeration or coalescence with SG attain narrower SDs than those formed solely by agglomeration, consistent with Spicer et al. [143].

narrows to $\sigma_{g, m}=1.84$ and 1.73 , respectively, approaching the so-called quasi-self-preserving limit of that regime consistent again with Goudeli et al. [125]. When SG takes place also (Fig. 4b), the primary particle size increases rapidly (Fig. 3b) while the $\sigma_{g, m}=1.33$ at $t=10 \mathrm{~ms}$ is well below the above SPSD limit for pure agglomeration, as ongoing SG narrows the SD [149]. Continuing agglomeration, however, broadens the SD with the formation of larger agglomerates, increasing gradually $\sigma_{g, m}$ to 1.42 and 1.53 at $\mathrm{t}=20$ and $30 \mathrm{~ms}$, respectively. These SDs are narrower than those expected for agglomerates growing solely by coagulation as SG narrows their SD well below the SPSD limit of 2.03 [125].

Coalescence between nascent soot particles (Fig. 4c) shifts the SD to smaller mobility sizes than in its absence (Fig. 4b) and further reduces $\sigma_{g, m}$ to 1.14 at $t=10 \mathrm{~ms}$ due to the dominance of SG. Coagulation is slower when full coalescence takes place upon collision, as smaller collision diameters lead to fewer collisions in the free molecular regime [131]. The average size increases only up to $15 \mathrm{~nm}$ and $\sigma_{g, m}$ only up to 1.26 at $t=30 \mathrm{~ms}$, much lower than the self-preserving limit of 1.46 for coagulation of spheres in the free molecular regime [150]. This further indicates the dominance of SG at these short residence times. The SDs of nascent soot particles growing by agglomeration or full 
coalescence with SG (Fig. 4b, c) are narrower than the corresponding SDs in the absence of SG (Fig. 4a) and consistent with the $\mathrm{TiO}_{2}$ aerosol dynamics at high concentrations of Spicer et al. [143].

Figure 5 shows the evolution of mean mobility, $\bar{d}_{m}$ (solid lines), and primary particle, $\bar{d}_{p}$ (broken line), diameters and average number of primary particles per aggregate/agglomerate, $\bar{n}_{p}$ (dotted lines, right-hand axis), of soot growing by agglomeration without (green lines) and with SG (blue lines) and coalescence (red line). Agglomeration of newly-incepted soot particles with $\bar{d}_{p}=2 \mathrm{~nm}$ and without any coalescence or SG increases $\bar{d}_{m}$ rapidly up to $60 \mathrm{~nm}$ and $\bar{n}_{p}$ up to $2^{\prime} 000$ at $t \approx 40 \mathrm{~ms}$, producing large ramified structures (Fig. 3a).

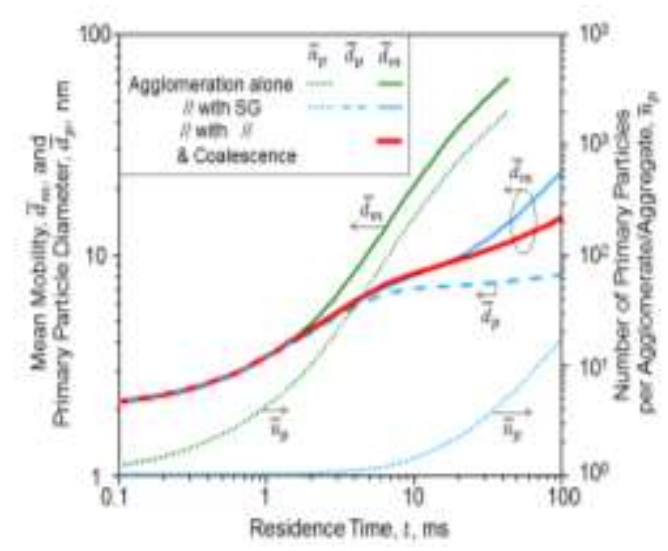

Figure 5. Evolution of mean $\bar{d}_{m}$ (solid lines), primary particle diameter, $\bar{d}_{p}$ (broken lines), and $\bar{n}_{p}$ (right-hand axis, dotted lines) of soot particles growing by agglomeration with (blue lines) or without SG (green lines) and full coalescence with SG (red line). As a reaction-limited mechanism, SG leads to more compact structures of smaller sizes and surface areas than the diffusion-limited agglomeration (DLA) mechanism, consistent with Kolb and Jullien [148]. In the presence of acetylene molecules, the evolution of $\bar{d}_{m}$ by agglomeration or full coalescence are identical up to $t=4$ ms and $\bar{d}_{m}<8 \mathrm{~nm}$, as particles are in the coalescing regime [113] and grow mainly by SG, keeping their spherical shape. When agglomeration becomes dominant $(t>15 \mathrm{~ms}), \bar{n}_{p}$ and $\bar{d}_{m}$ increase further compared to full coalescence. At $t=$ $100 \mathrm{~ms}$, the $\bar{d}_{m}$ of soot aggregates is $40 \%$ larger than the corresponding size of a sphere.

When SG takes place, necks start to grow between primary particles and the resulting soot aggregates are much smaller than the agglomerates made without SG at the same time (see also Fig. 3a, b), reaching $\bar{d}_{m}=24$ nm (solid lines) and $\bar{n}_{p}=18$ (dotted lines) at $t=100 \mathrm{~ms}$. As a reaction-limited particle growth mechanism, SG leads to more compact structures of smaller mobility and gyration diameters, consistent again with Kolb and Jullien [148]. When SG takes place, the $\bar{d}_{m}$ evolution by agglomeration with or without coalescence are identical up to 2 ms, since agglomeration is too slow for such small particles and SG dominates conserving their spherical shape $\left(\bar{d}_{m} \approx \bar{d}_{p}\right)$. Later on $(2<t<15 \mathrm{~ms})$, both agglomeration and SG dominate leading to the formation of small soot aggregates as seen in Fig. $3 \mathrm{~b}$ (e.g. at $10 \mathrm{~ms}$ ). 
As SG ends $(t \approx 10 \mathrm{~ms}), \bar{d}_{p}$ levels off at about $7 \mathrm{~nm}$ while $\bar{n}_{p}$ and $\bar{d}_{m}$ increase even more compared to full coalescence (red solid line). At $t=100 \mathrm{~ms}$, the $\bar{d}_{m}$ of soot aggregates is $40 \%$ larger than that of equivalent spherical particles.

Figure 6 shows the evolution of $\sigma_{g, m}$ of soot particles growing by full coalescence with (red line) and without SG (purple lines), as well as agglomeration with (blue line) and without SG (green lines). Without SG, particles coagulating in the free molecular regime with or without coalescence (e.g. agglomeration) rapidly reach their self-preserving $\sigma_{g, m}=$ 1.46 for spheres [150; purple dotted line] or 2.03 for agglomerates [125; green dot-broken line], respectively. In the absence of coalescence (pure agglomeration; green line) for $\bar{d}_{m}>20 \mathrm{~nm}(t>10 \mathrm{~ms}$ ), the polydispersity of agglomerate SD decreases since agglomerate growth by coagulation enters the transition regime approaching a quasi-SPSD with a minimum $\sigma_{g, m}=1.6$ at mobility-based diffusive Knudsen number, defined as the ratio of the particle stopping distance, $\lambda_{a}$, to the particle mobility size, $K n_{D, m}=2 \lambda_{d} / d_{m}=0.92$, consistent with Goudeli et al. [125].

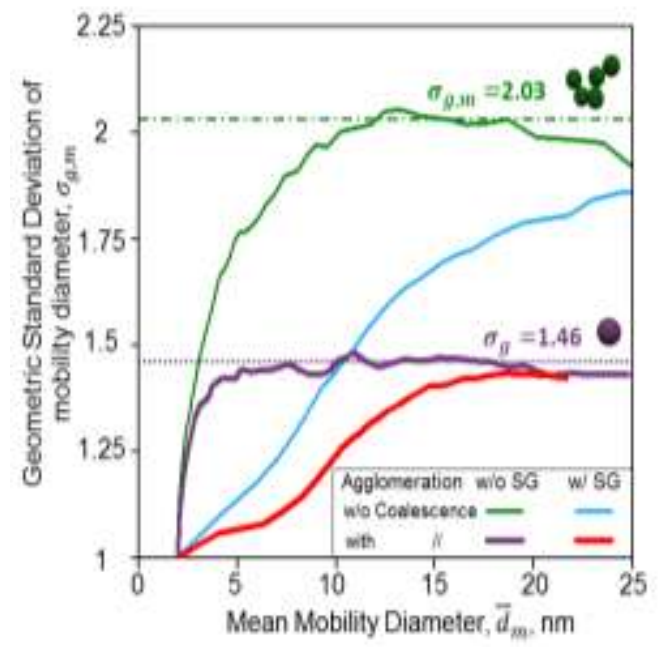

Figure 6. Evolution of mobility-based geometric standard deviation, $\sigma_{g, m}$, of soot particles growing by full coalescence with (red line) and without SG (purple lines), as well as agglomeration with (blue line) and without SG (green lines). In the absence of SG, particles coagulating by full coalescence or agglomeration reach rapidly their self-preserving $\sigma_{g, m}=$ 1.46 and $\sigma_{g, m}=2.03$ for spheres [150] and agglomerates [125], respectively, in the free molecular regime. For $\bar{d}_{m}>20$ $\mathrm{nm}, \sigma_{g, m}$ of agglomerate size distribution drops since the particle growth enters the transition regime. SG narrows the particle size distribution and delays the attainment of the asymptotic limit for both full coalescence (blue line) and agglomeration (red line), consistent with Spicer et al. [143]. For example, aggregates of $\bar{d}_{m}=8 \mathrm{~nm}$ formed by agglomeration and SG have $\sigma_{g, m}=1.3$, while agglomerates of the same $\bar{d}_{m}$ have almost reached their self-preserving $\sigma_{g, m}$ of 2.03. Full coalescence in the presence of SG is commonly assumed for particles of sizes below $20 \mathrm{~nm}$ [119, 120, 151]. Neglecting the fractal nature of soot particles, however, leads to underestimation of their polydispersity up to $40 \%$.

The SG narrows temporarily the particle size distribution for agglomeration with (red line) and without coalescence (blue line), consistent with Spicer et al. [143]. For example, aggregates of $\bar{d}_{m}=8 \mathrm{~nm}$ formed by SG and agglomeration without coalescence (blue line) have $\sigma_{g, m}=1.3$, while agglomerates of primary particles in point contact (green line) have $\sigma_{g, m}=1.92$. Full coalescence further reduces $\sigma_{g, m}$ to 1.14 at the same time. As soot particles become 
larger, the effect of surface growth on size distribution wanes. Similar to the dynamics of spherical particles by coagulation and surface growth [143], the evolutions of $\sigma_{g, m}$, by surface growth and coagulation with (red line) and without coalescence (blue line) reach their self-preserving values by coagulation alone (purple and green lines, respectively). Typically, coalescence is considered the dominant growth mechanism for soot particles smaller than $20 \mathrm{~nm}$ in many models $[119,120,151]$. Neglecting, however, the fractal nature of soot particles, leads to non-trivial underestimation of the polydispersity of the mobility size, e.g. here about $40 \%$ at the $95 \%$ of the confidence interval for $\bar{d}_{m}=20 \mathrm{~nm}$.

\subsection{Comparison with experiments}

Figure 7 shows the evolution of DEM-derived mass fractal dimension, $D_{f}$ (solid line), and mass-mobility exponent, $D_{f m}$ (dot-broken line), of nascent soot particles growing by agglomeration and SG at the flame conditions of Schenk et al. [117] as a function of mean mobility diameter, $\bar{d}_{m}$, using Eqs. (6) and (7), respectively. The evolution of $D_{f}$ is compared to that obtained from microscopy measurements of Schenk et al. [117; symbols, insets in Fig. 7] at 0.5 (triangles), 0.8 (circles) and $1.2 \mathrm{~cm}$ (squares) Height Above the Burner (HAB). The microscopy images of aggregates with $d_{m}>12 \mathrm{~nm}$ [117] are in good agreement with height and phase imaging by Atomic Force Microscopy [152], indicating that in this size range nascent soot morphology is not distorted upon impact on the substrate allowing quantitative comparisons with the present DEM. The relationship of Lee and Kramer [153: Eq. (16)] is used to convert the peripheral fractal dimension, $D_{p f}$, as reported in Schenk et al. [117; symbols, insets in Fig. 7] to $D_{f}$.

The images and $D_{f}$ of Schenk et al. [117; insets and symbols in Fig. 7] are similar to representative DEMobtained blue images and $D_{f}$ of soot particles of the same $\bar{d}_{m}$ in Fig. 7. Soot particles are quite spherical for $\bar{d}_{m}<6 \mathrm{~nm}$ ( $t<4 \mathrm{~ms}$ ) where SG is dominant, consistent with the simulations of Mitchell and Frenklach [113] and experiments of Schenk et al. [117]. When SG ends $\left(\bar{d}_{m}>10 \mathrm{~nm}\right)$ and particle growth by agglomeration of existing soot particles prevails, $D_{f}$ and $D_{f m}$ start to decrease as more open and rather elongated structures are formed by DEM (insets for $\bar{d}_{m}=$ $14,18$ and $22 \mathrm{~nm})$. As the number density of nascent soot decreases, cluster-cluster agglomeration prevails and $D_{f m}$ decreases more slowly than $D_{f}$ that eventually drops below $D_{f m}$, since larger agglomerates are gradually formed approaching their asymptotic fractal-like values. The difference between $D_{f m}$ and $D_{f}$ stems from their differing asymptotic values of $2.17 \pm 0.1$ [136] and $1.91 \pm 0.03$ [135], respectively, for ballistic cluster-cluster agglomeration, which is equivalent to coagulation in the free molecular regime here. Furthermore, the evolution of $D_{f m}$ is less sensitive to 
formation of chemical bonds between soot primary particles compared to the respective evolution of $D_{f}$, consistent with the sintering simulations of Eggersdorfer et al. [154: Fig. 6].

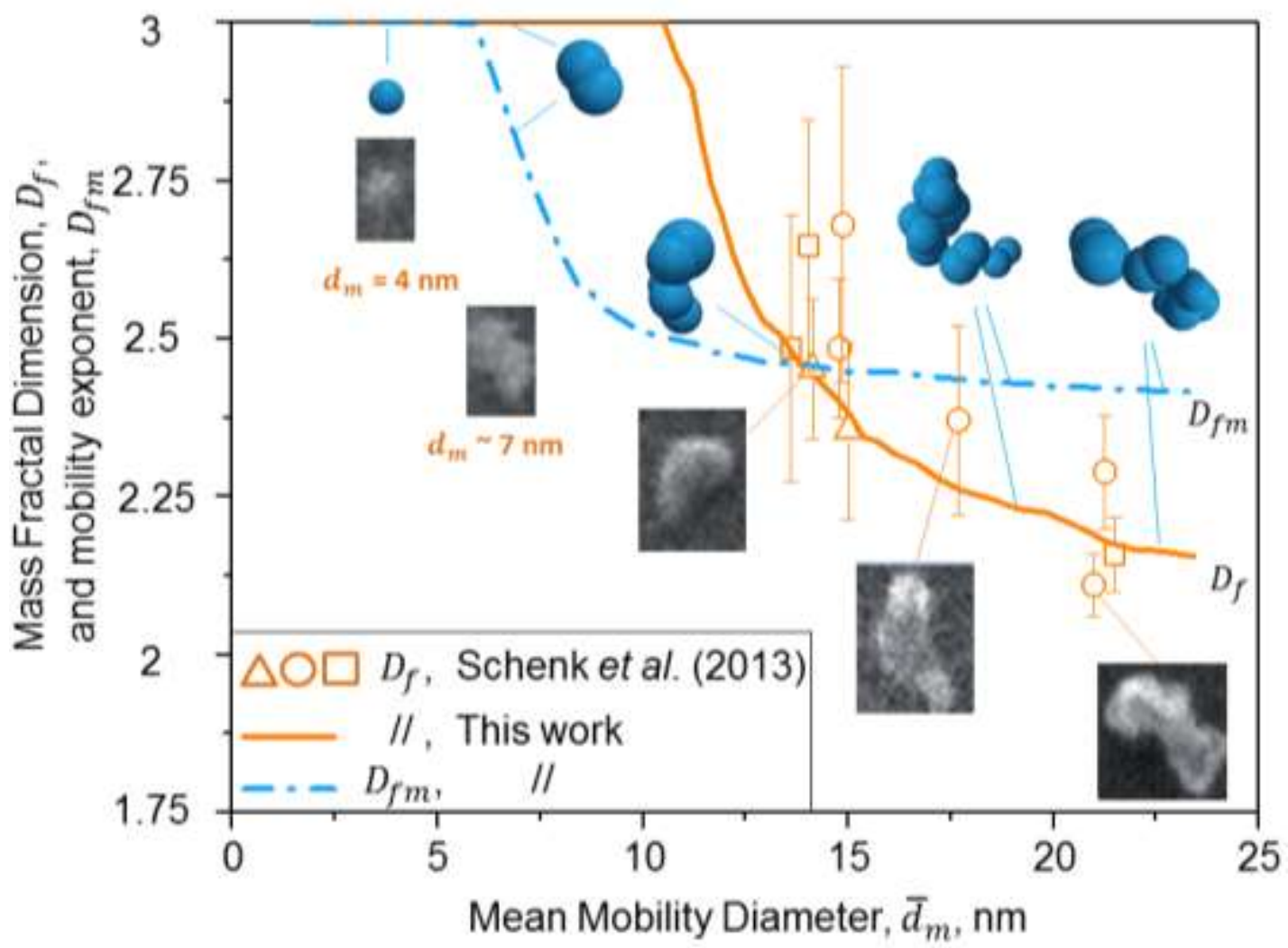

Figure 7. Evolution of DEM-derived $D_{f}$ (solid line) and $D_{f m}$ (dot-broken line) of soot particles growing by agglomeration with SG as a function of $\bar{d}_{m}$. The DEM-derived $D_{f}$ is in excellent agreement with experiments of Schenk et al. [117; symbols, insets] at HAB = 0.5 (triangles), 0.8 (circles) and $1.2 \mathrm{~cm}$ (squares). Soot particles remain spherical for $\bar{d}_{m}<6 \mathrm{~nm}(t<4 \mathrm{~ms})$ where SG is dominant, consistent with Schenk et al. [117]. When agglomeration prevails, aggregates are formed so $D_{f}$ and $D_{f m}$ start to decrease. The formed aggregates have much more compact structures than those corresponding to the asymptotic limit of $D_{f}=1.91 \pm 0.03$ [135] and $D_{f m}=2.17 \pm 0.1$ [136] for agglomerates in the free molecular regime.

The primary particles of the resulting aggregates can hardly be discerned from microscopy and DEM-obtained images (insets), as new soot layers are formed on their surface by acetylene surface reaction and bury the original primary particle boundaries. Here, both DEM- and microscopy-obtained aggregates have much more compact structures than those corresponding to their asymptotic limits of agglomerates in the free molecular regime [135,136], but they are more open than those obtained by Mitchell and Frenklach [113] by particle-cluster ballistic agglomeration. The DEMderived $D_{f}$ is in good agreement with the experiments of Schenk et al. [117], pointing out the capacity of the present DEM (solid line) to follow closely the nascent soot dynamics by surface growth with concurrent particle-cluster and cluster-cluster agglomeration.

Figure 8 shows the DEM-derived regression (blue line) of the scaling law (Eq. (7)) between aggregate mass, $m$, and $d_{m}$ of soot growing by agglomeration and SG for the conditions of Fig. 7 [117] with $\bar{d}_{m}=15.1 \mathrm{~nm}$ (solid line) 
corresponding to $\mathrm{HAB}=1.2 \mathrm{~cm}$. The DEM-derived particle mass and mobility size distribution corresponds to $\mathrm{HAB}=$ $1.2 \mathrm{~cm}$ at $t=45 \mathrm{~ms}$ based on the measured $\bar{d}_{m}$ of Schenk et al. [117] and the residence time computed for HAB $=1.2 \mathrm{~cm}$ by Abid et al. [155]. The slope of the power law (Eq. (7)) corresponds to $D_{f i n}=2.47$, slightly higher than the corresponding $D_{f}=2.45$.

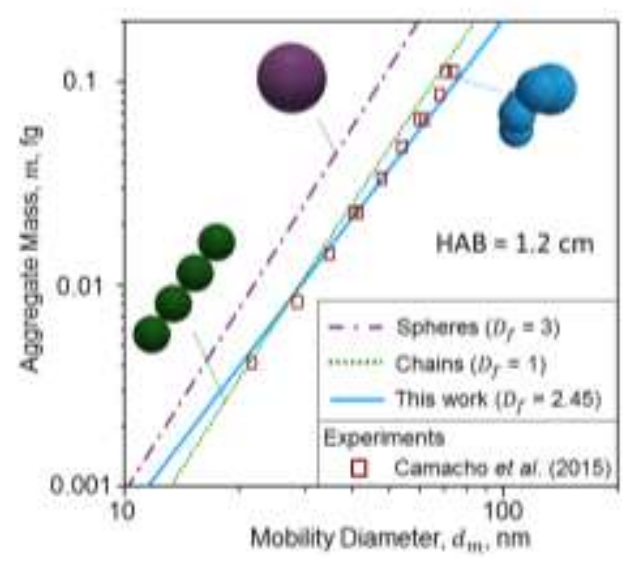

Figure 8. DEM-derived power law (solid line) of aggregate mass, $m$, and $d_{m}$ are in good agreement with mass-mobility measurements of Camacho et al. [4; symbols] at $\mathrm{HAB}=1.2 \mathrm{~cm}$. Models of spheres (dot-broken line) and straight chains of four primary particles [4; dotted line] overestimate the mass of nascent soot aggregates nearly 3 times and $20 \%$, respectively, for $d_{m}=60 \mathrm{~nm}$.

The DEM-derived relationship between nascent soot mass and mobility diameter is compared to the massmobility measurements of Camacho et al. [4; squares] at $\mathrm{HAB}=1.2 \mathrm{~cm}$. The DEM is in good agreement with the experiments of Camacho et al. [4] for $m>0.01 \mathrm{fg}$. The small discrepancy at $m<0.01 \mathrm{fg}$ can be attributed to the low resolution of mass measurements for so small nascent soot particles. The reduction of the DEM-derived $D_{f m}$ to 2.47 (solid line) quantifies the delicate changes of nascent soot structure up to $\mathrm{HAB}=1.2 \mathrm{~cm}$, as the aggregates become less compact by particle- and cluster-cluster collisions, consistent with the low effective densities of $0.7 \mathrm{~g} / \mathrm{cm}^{3}$ obtained by Camacho et al. [4] at $\mathrm{HAB}=1.2 \mathrm{~cm}$. The spherical model for nascent soot morphology (dot-broken line) leads to nearly 3 times overestimation of the aggregate mass for $d_{m}=60 \mathrm{~nm}$. Camacho et al. [4] described this evolution of massmobility relationship by various fixed geometrical models of soot morphology. The straight chain composed of 4 primary particles in point contact (green dotted line in Fig. 8) was the most successful, overestimating the nascent soot mass up to $20 \%$ for $d_{m}=60 \mathrm{~nm}$. This difference could be attributed to the low $D_{f}=1$ of chains. The DEM-obtained aggregates have also elongated structures at $\mathrm{HAB}=1.2 \mathrm{~cm}$, similarly to a 4-particle straight chain. The $\mathrm{SG}$-induced chemical bonding between primary particles, however, increases $D_{f}$ to larger values $(\sim 2.45)$ than the well-known value of $D_{f}=1$ for straight chains. This is in qualitative agreement with Oh and Sorensen [156], who showed that the $D_{f}$ of aggregates increases as a function of the overlap between primary particles. This is similar to the chemical bonding of primary particles induced by surface growth studied here. Thus, the present DEM seems to demonstrate quite realistically the evolution of nascent soot 
$\bar{n}_{p}, D_{f}$ and $D_{f m}$ by consistent agglomeration and surface growth particle dynamics $[113,143,151]$ achieving good agreement with different experimental methods and data $[4,117]$.

Figure 9 shows the DEM-derived mobility size distributions of soot particles growing by agglomeration with (blue line) or without SG (green line) and full coalescence with SG (red line) at $1.2 \mathrm{~cm} \mathrm{HAB}$ in comparison to microscopic [117; triangles] and mobility size measurements [4; squares]. The DEM-derived size distribution of soot growing by agglomeration and SG (blue line) is in excellent agreement with both microscopic [117; triangles] and mobility size measurements [4; squares]. The distribution has $\sigma_{g, m}=1.65$, narrower than the SPSD limit of 2.03 in the free molecular regime [125], since the agglomerates grow in the transition regime at this HAB. As a reaction-limited mechanism, SG (red and blue lines) leads to smaller mobility diameters than agglomeration alone (green line) that leads to formation of agglomerates having $\bar{d}_{m}=63.1 \mathrm{~nm}$ at $\mathrm{HAB}=1.2 \mathrm{~cm}(t=45 \mathrm{~ms})$, more than thrice larger than the soot aggregate mobility diameter measured by Schenk et al. [117; triangles] and Camacho et al. [4; squares]. When agglomeration with full coalescence takes place along with SG (red line), it further reduces $\bar{d}_{m}$ to $11.6 \mathrm{~nm}$ and underestimates the measured mean mobility diameter (symbols) by $50 \%$. The size distribution of soot growing by full coalescence and SG (red line) has $\sigma_{g, m}=1.35$ and is narrower than the measured ones (symbols), highlighting the need to account for the evolving soot structure during particle dynamics.

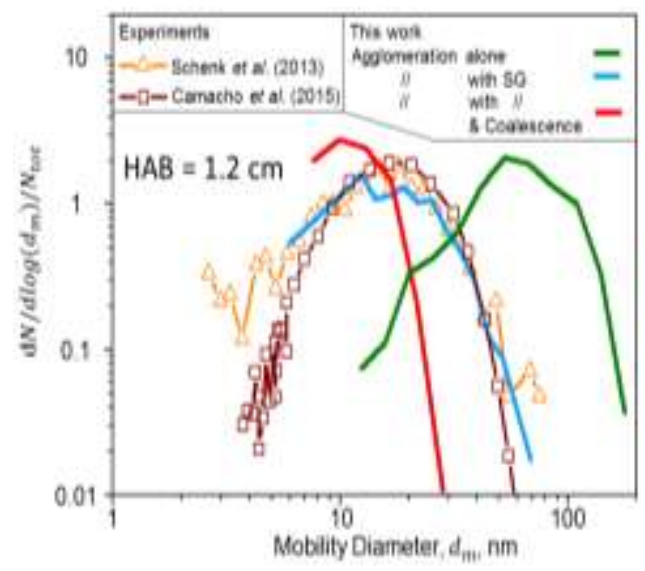

Figure 9. DEM-derived mobility size distributions of soot particles growing by agglomeration with (blue line) or without $\mathrm{SG}$ (green line) and full coalescence with SG (red line) at $\mathrm{HAB}=1.2 \mathrm{~cm}(t=45 \mathrm{~ms})$ in comparison to microscopic [117; triangles] and mobility size measurements [4; squares]. Agglomeration with SG is in excellent agreement with experimental data (symbols). In contrast, neglecting SG (green line) or agglomeration (by considering full coalescence, red line) substantially overpredicts or underpredicts, respectively, the experimental data.

\subsection{Effect of acetylene concentration on soot morphology and size distribution}

The hydrogen atom concentration is varied during soot growth by agglomeration and SG in order to attain fixed maximum volume fraction, $f_{v, \max }$, of $5 \cdot 10^{-9}, 10^{-8}$ (shown in Figs. 2-9) and $10^{-7}$. Higher $f_{v, \max }$ are associated with increasing equivalence ratio, $\varphi[157]$. 
Figure 10 shows snapshots of an exemplary soot particle with initial primary particle diameter $d_{p, o}=2 \mathrm{~nm}$ growing by agglomeration with SG attaining $f_{v, \max }$ of (a) $5 \cdot 10^{-9}$, (b) $10^{-8}$ (identical to Fig. 3b) and (c) $10^{-7}$ at $t=10,20$ and $30 \mathrm{~ms}$. At $t$ $=10 \mathrm{~ms}, \mathrm{SG}$ is dominant for all three $f_{v, \max }$ forming compact soot dimers of increasing primary particle size and keeping the average number of primary particles per aggregate, $\bar{n}_{p}$, below 2 . However, increasing $f_{v, \max }$ by one order of magnitude (Fig. 10a and c) leads to larger neck sizes (compared to primary particle size) and subsequently stronger degree of chemical bonding between primary particles and thus more compact structures.

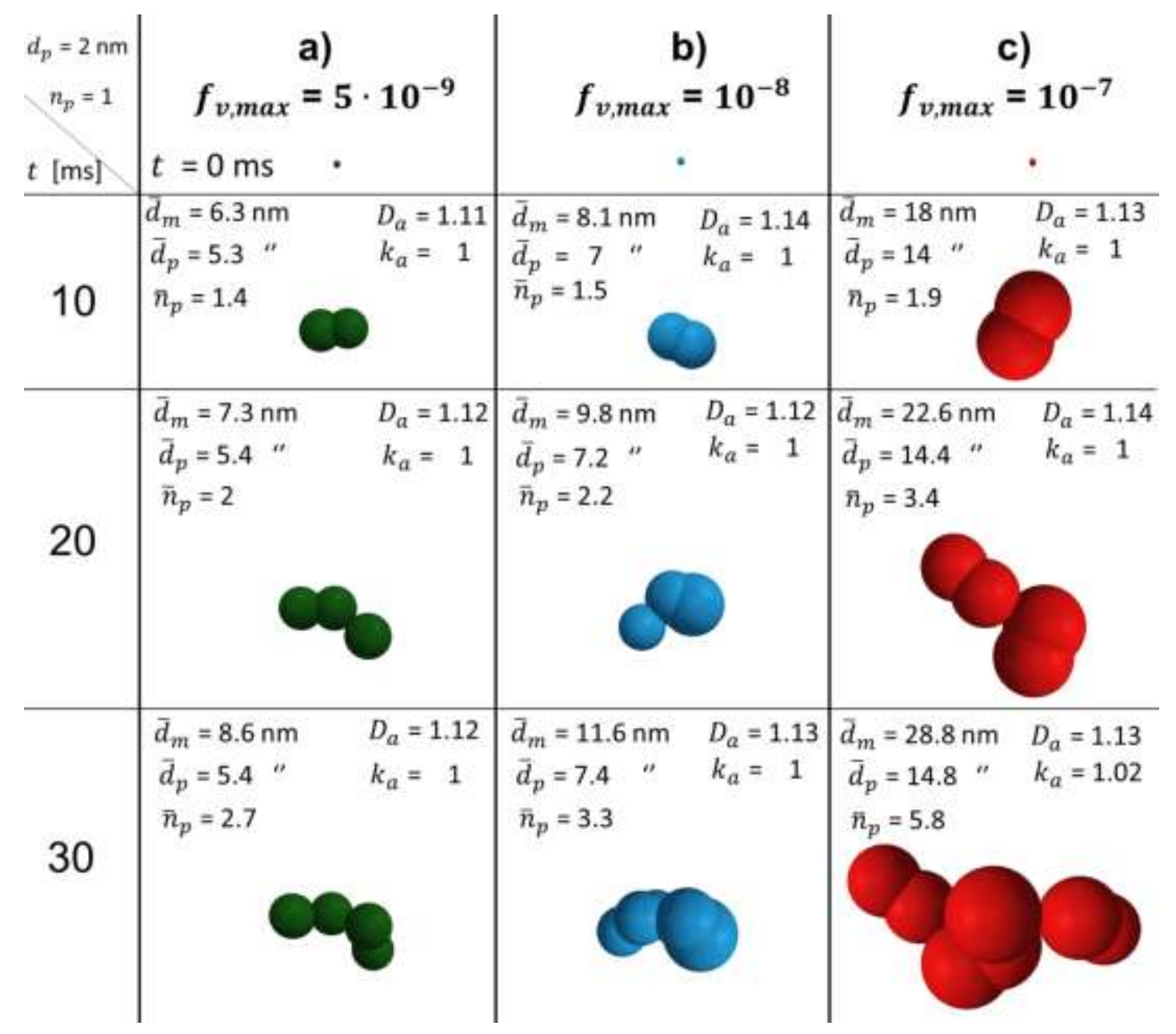

Figure 10. Snapshots of soot particles growing by agglomeration and SG attaining maximum volume fraction, $f_{v, \text { max }}$ of (a) $5 \cdot 10^{-9}$, (b) $10^{-8}$ and (c) $10^{-7}$. At $t=10 \mathrm{~ms}$, SG is dominant for all three $f_{v \text {, max }}$ forming compact soot dimers and keeping the average number of primary particles per aggregate, $\bar{n}_{p}$, below 2 . For $t \geq 20 \mathrm{~ms}$, agglomeration gradually takes over and increases $\bar{n}_{p}$. The average primary particle diameter, $\bar{d}_{p}$, grows effectively up to $t=10 \mathrm{~ms}$ and levels off after 20 ms. Higher $f_{v, \max }$ lead to larger primary particles. Soot aggregates attaining $f_{v, \max }=10^{-7}$ have large $\bar{d}_{p}$ and coagulate fast leading to larger $\bar{d}_{m}$ and $\bar{n}_{p}$ than those formed for $f_{v, \max }=5 \cdot 10^{-9}$ and $10^{-8}$ at the same $t$. The projected area exponent, $D_{a}$, and prefactor, $k_{a}$, of small nascent soot aggregates up to $30 \mathrm{~nm}$ does not vary significantly with $f_{v, \max }$, having average $D_{a}$ $=1.13 \pm 0.02$ and $k_{a}=1 \pm 0.01$.

This is consistent with the mass-mobility measurements of Ghazi et al. [158] who also obtained more compact soot aggregates with increasing $\varphi\left(\right.$ and $\left.f_{v, \max }\right)$ from an inverted laminar diffusion flame. For $t \geq 20$ ms, agglomeration 
gradually takes over and increases $\bar{d}_{m}$ and $\bar{n}_{p}$. The average primary particle diameter, $\bar{d}_{p}$, grows quickly up to $t=10 \mathrm{~ms}$ and levels after that. Higher $f_{v, \max }$ lead to larger and more polydisperse primary particles, as also seen in Fig. 3b. Soot aggregates made at $f_{v, \max }=10^{-7}$ have large $\bar{d}_{p}$ and coagulate fast leading to larger $\bar{d}_{m}$ and $\bar{n}_{p}$ than at lower $f_{v, \max }$ at the same $t$, as expected by a mass balance and coagulation theory.

The projected area exponent, $D_{a}$, and prefactor, $k_{a}$, derived from Eq. (10) do not depend on $f_{v, \max }$ for small nascent soot aggregates with $\bar{d}_{m}$ up to $30 \mathrm{~nm}$, yielding average values of $D_{a}=1.13 \pm 0.02$ and $k_{a}=1 \pm 0.01$.

This DEM-derived $D_{a}$ for agglomeration and SG is larger than the respective value of 1.07 for sintering [71]. Surface growth increases the aggregate surface (and projected) area by new soot formation, in contrast to sintering where that area steadily decreases as mass is conserved [159]. The nascent soot primary particle size can be estimated from massmobility measurements, using the scaling law of Eggersdorfer et al. [71]:

$d_{p}=\left(\frac{\pi k_{a}}{6 v}\left(d_{m}\right)^{2 D_{a}}\right)^{1 /\left(2 D_{a}-3\right)}$

in tandem with the DEM-derived $D_{a}$ and $k_{a}$ for agglomeration and SG (Eq. (12)). Following this procedure with the massmobility data of Camacho et al. [4; Fig. 8] a mean $\bar{d}_{p}$ of $10 \mathrm{~nm}$ at both HAB is obtained, in good agreement with the DEM-derived $\bar{d}_{p}=7.5 \mathrm{~nm}$ at the same flame conditions (Fig. 5; broken line).

Figure 11 shows the DEM-derived mobility SDs of soot growing by agglomeration with SG attaining $f_{v, \max }$ of (a) $5 \cdot 10^{-9}$, (b) $10^{-8}$ (identical to Fig. 4b) and (c) $10^{-7}$ at $t=10$ (dotted lines), 20 (dot-broken lines) and $30 \mathrm{~ms}$ (solid lines). The aggregate SD shifts to larger mobility diameters with increasing $f_{v, \text { max }}$, as the enlargement of primary particle size enhances the coagulation rate (Fig. 10). Soot aggregates formed for $f_{v, \max }=5 \cdot 10^{-9}$ and $10^{-8}$ have similar $\bar{d}_{m}$ and $\bar{d}_{p}$, and thus the width of their SDs do not differ significantly. For example, at $t=30 \mathrm{~ms}$ aggregates with $f_{v, \max }=5 \cdot 10^{-9}$ have $\sigma_{g, m}=$ 1.49, while those with $f_{v, \max }=10^{-8}$ have $\sigma_{g, m}=1.53$. Increasing further mobility and primary particle sizes at $f_{v, \max }=10^{-7}$, accelerates coagulation and broadens the SD which is still below its asymptotic self-preserving geometric standard deviation of 2.03 in the free molecular regime [125].

Figure 12a shows the evolution of $\bar{d}_{m}$ (solid lines) and $\bar{d}_{p}$ (broken lines) of soot aggregates growing by agglomeration and SG attaining $f_{v, \text { max }}=5 \cdot 10^{-9}$ (green lines), $10^{-8}$ (blue lines) and $10^{-7}$ (red lines). For all three $f_{v, \text { max }}$, SG conserves initially the spherical shape of soot aggregates keeping $\bar{d}_{m} \approx \bar{d}_{p}$, as also seen in Fig. 5. The time at which the hydrogen atoms needed for acetylene SG are consumed and SG ends, the $\bar{d}_{p}$ levels off indicating when agglomeration 
starts to dominate. This time is increasing from 2 to $8 \mathrm{~ms}$ for decreasing $f_{v, \max }$ from $10^{-7}$ to $5 \cdot 10^{-9}$, respectively, in agreement with Balthasar and Frenklach [160], who showed that the transition to the aggregation growth regime happens at lower residence times with increasing $\varphi$ and thus $f_{v, \max }$. The asymptotic $\bar{d}_{p}$ increases from 5.5 for $f_{v, \max }=5 \cdot 10^{-9}$ to about $15 \mathrm{~nm}$ for $f_{v, \text { max }}=10^{-7}$. The aggregates formed at higher $f_{v, \text { max }}$ have larger $\bar{d}_{p}$ and agglomerate faster attaining larger $\bar{d}_{m}$, consistent with the SMPS measurements of Maricq et al. [115] in laminar premixed ethylene flames of different $\varphi$ and subsequently $f_{v, \max }$ here.

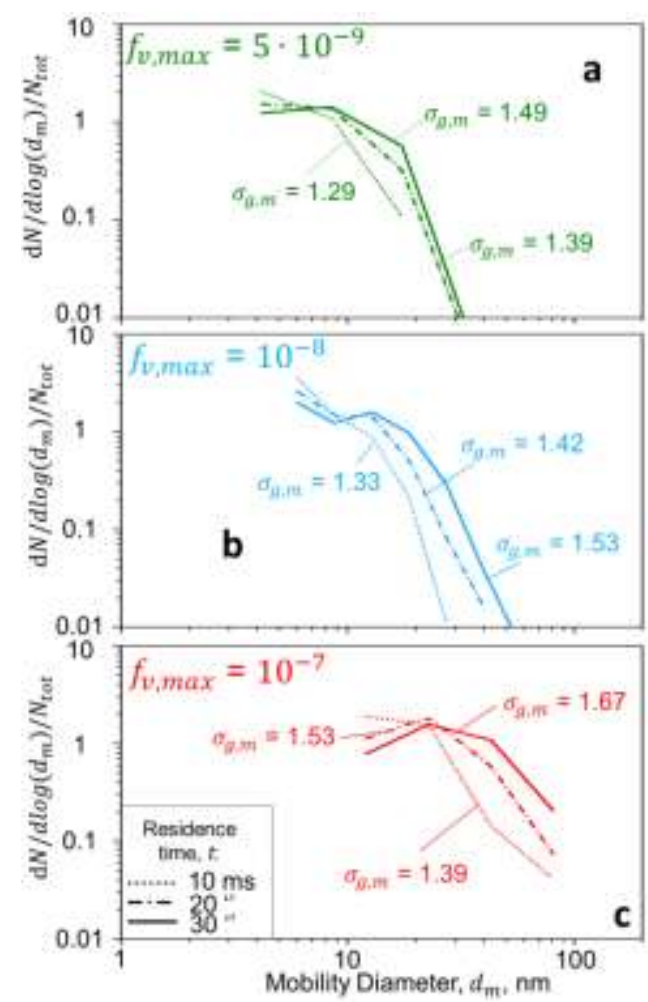

Figure 11. DEM-obtained mobility size distributions (SDs) of soot particles growing by agglomeration and SG attaining $f_{v, \text { max }}$ of (a) $5 \cdot 10^{-9}$, (b) $10^{-8}$ and (c) $10^{-7}$ at $t=10$ (dotted lines), 20 (dot-broken lines) and (c) $30 \mathrm{~ms}$ (solid lines). The aggregate SD shifts to larger mobility diameters with increasing $f_{v, \max }$. For $f_{v, \max }=10^{-7}$ (Fig. 11c) much larger primary particles are formed enhancing coagulation rate and thus broadening faster the SD.

Figure $12 \mathrm{~b}$ shows the evolution of $\sigma_{g, m}$ (solid lines) and the geometric standard deviation of primary particle diameter, $\sigma_{g, p}$ (broken lines), of soot aggregates growing by agglomeration and SG. For $f_{v, \max }=5 \cdot 10^{-9}$ (green lines) and $10^{-8}$ (blue lines) particles grow solely in the free molecular regime increasing monotonically $\sigma_{g, m}$ up to 1.8 within this residence time. The contribution of SG in the formation of nascent soot aggregates of the same $\bar{d}_{m}$ is increasing with higher $f_{v, \text { max }}$, reducing both $\sigma_{g, m}$ and $\sigma_{g, p}$. In terms of residence time (Fig. 12c), however, more polydisperse mobility size distributions are obtained in the free molecular regime for increasing $f_{v, \text { max }}$ from $5 \cdot 10^{-9}$ to $10^{-7}$, since agglomeration is accelerated with larger mobility and primary particle diameters (Fig. 12a) broadening the SD. This is consistent with the simulations of Tsantilis and Pratsinis [161] for spheres and the SMPS measurements of Maricq et al. [115]. This 
enhancement of $\sigma_{g, m}$ could be attributed to the larger $\bar{d}_{p}$ attained by the surface reaction of acetylene that leads to faster agglomeration.
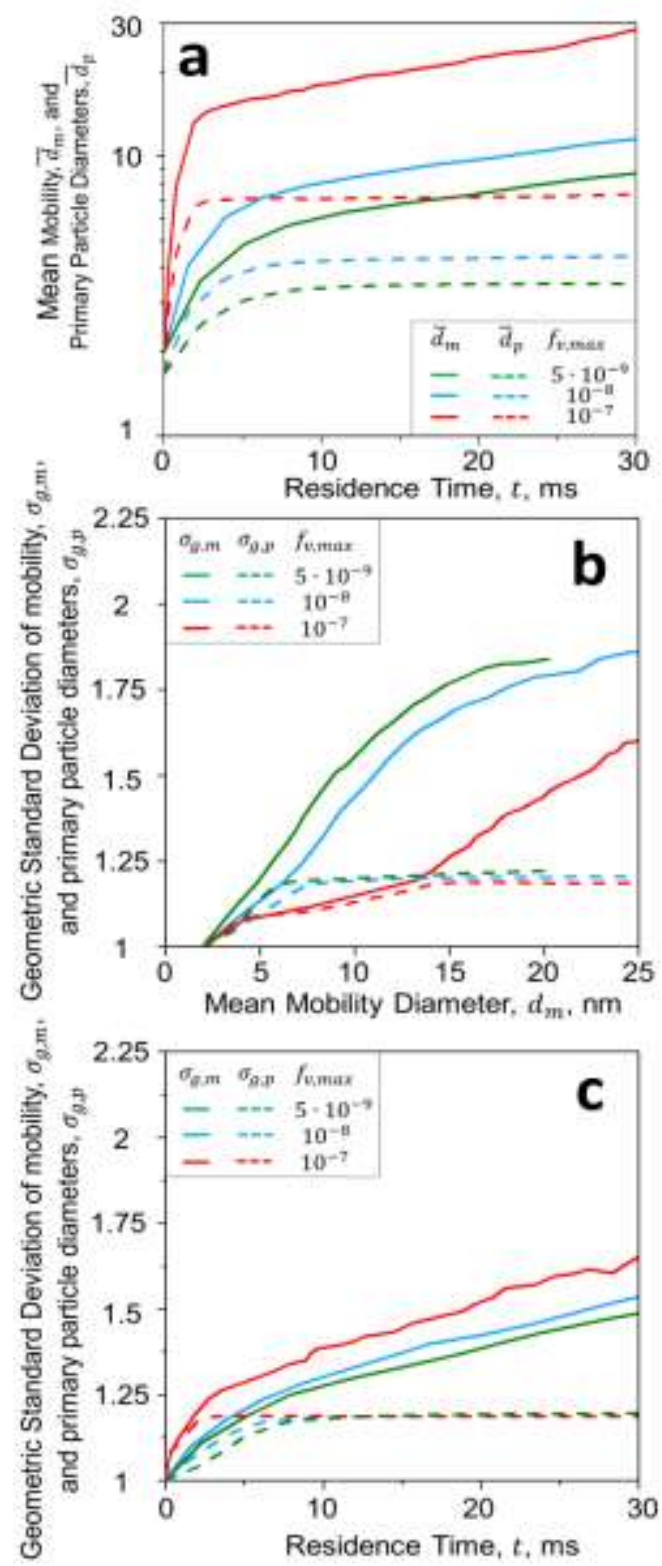

Figure 12. (a) Evolution of mean mobility, $\bar{d}_{m}$ (solid lines), and primary particle diameter, $\bar{d}_{p}$ (broken lines), of soot particles growing by agglomeration and SG attaining $f_{v, \text { max }}$ of $5 \cdot 10^{-9}$ (green lines), $10^{-8}$ (blue lines) and $10^{-7}$ (red lines). When SG is dominant, soot aggregates remain compact (Fig. 10) with $\bar{d}_{m} \approx \bar{d}_{p}$ for all three $f_{v, \max }$. The time at which agglomeration takes over with $\bar{d}_{m}$ becoming larger than $\bar{d}_{p}$ decreases from about 8 to 2 ms for increasing $f_{v, \max }$, consistent with the simulations of Balthasar and Frenklach [160]. Larger primary particles made for higher $f_{v, \text { max }}$ agglomerate faster inducing the formation of aggregates of larger $\bar{d}_{m}$. (b, c) Evolution of mobility, $\sigma_{g, m}$ (solid lines), and primary particle diameter geometric standard deviation, $\sigma_{g, p}$ (broken lines), of soot growing by agglomeration and SG attaining $f_{v, \text { max }}$ of $5 \cdot 10^{-9}$ (green lines), $10^{-8}$ (blue lines) and $10^{-7}$ (red lines) in terms of (b) $\bar{d}_{m}$ and (c) $t$. The contribution of SG in the formation of nascent soot aggregates of the same $\bar{d}_{m}$ is increasing with higher $f_{v, \max }$, reducing both $\sigma_{g, m}$ and $\sigma_{g, p}$. However, larger primary particles formed for higher $f_{v, \max }$ (a) collide faster increasing $\sigma_{g, m}$ and $\sigma_{g, p}$ as a function of time, in agreement with experiments $[115,116]$. 
As $\bar{d}_{m}$ and $\bar{n}_{p}$ increase by agglomeration, the necks induced by SG do not grow uniformly between the primary particles (Fig. 10) enhancing $\sigma_{g, p}$. A nearly asymptotic $\sigma_{g, p}$ of about 1.18 for all $f_{v, \max }$ is attained, in agreement with the average $\sigma_{g, p}$ of 1.15 measured from microscopy images of mature soot in laminar premixed flames of different $\varphi$ (or $f_{v, \max }$ here) by Oltmann et al. [116]. The DEM-derived $\sigma_{g, p}$ indicates that mature soot primary particle polydispersity originates from the primary particle size distribution of the nascent soot aggregates determined by agglomeration and SG.

\section{Conclusions}

In flame synthesis of materials there is keen interest on product "soot" or particle size, composition and morphology, in stark contrast to combustion research in connection to energy generation that treats soot as nuisance or pollutant. Today there is a good understanding of flame synthesis of simple oxides and nanostructured carbon to routinely manufacture commodities such as carbon blacks, optical fibers, fumed oxides, white pigments and photocatalysts at several t/h. This proven scalability of combustion along with its capacity to form sophisticated particle morphologies and metastable compositions of high purity makes it quite attractive for material synthesis. So detailed measurements and computations have advanced our understanding on the formation of far more sophisticated materials of much higher value than the above commodities, predominantly by spray combustion.

The high temperature residence time and the interplay of surface growth, coagulation and sintering determine the characteristics (size, composition and morphology) of flame-made particles. The high particle concentrations during flame synthesis of materials lead to the rapid attainment of the well-known fractal-like structure and self-preserving size distribution (SPSD) by coagulation, greatly simplifying the accounting of particle dynamics facilitating, thus, their incorporation in fluid mechanics for efficient aerosol reactor design. Mesoscale and molecular dynamic models provide further insight down to the atomistic level of particle dynamics and assist the determination of material properties in the respective size scale (nano vs. bulk) in connection to these fluid-particle mechanics models contributing to the so-called multiscale process design. Such computational schemes facilitate the extraction of power laws describing the dynamics of fractal-like particle morphology as with nascent soot, a bona fide nanomaterial.

Here, the evolution of nascent soot size, polydispersity and structure by surface growth and agglomeration was investigated by mesoscale simulations using Discrete Element Modeling (DEM). It is shown that the common assumption of steadily spherical nascent soot particles can underestimate up to $40 \%$ their average mobility size and polydispersity up to $40 \%$ leading to an overestimation of their mass by nearly a factor of 3 . Soot aggregates formed by agglomeration and surface growth are much smaller and more compact than those made by agglomeration alone. Surface growth narrows the size distribution of soot agglomerates/aggregates. The DEM-derived evolution of soot structure in 
terms of mass fractal dimension, $D_{f}$, and mass-mobility exponent, $D_{f m}$, is in excellent agreement with microscopy and mass-mobility measurements in a benchmark burner-stabilized stagnation ethylene flame. The relationship between mass and mobility size of nascent soot aggregates is a product of particle- and cluster-cluster agglomeration, as well as of the chemical bonding between primary particles due to surface growth. Aggregates grow larger and attain more ramified structure by cluster-cluster agglomeration. However, the obtained $D_{f}$ and $D_{f m}$ accounting for surface growth (SG) are much higher than the respective asymptotic values for agglomerates in the free-molecular regime, indicating the impact of SG in forming compact particles.

The DEM-obtained mobility size distributions are also in good agreement with those measured by microscopy and mass-mobility measurements at different heights above the burner. This indicates that the evolution of nascent soot aggregate size distribution can be described accurately by agglomeration and surface growth when accounting for the detailed soot structure. Higher soot volume fractions obtained by larger ethylene and subsequently acetylene concentrations result in larger mobility and primary particle sizes, enhancing the coagulation rate and increasing the polydispersity of both mobility and primary particle size distribution. The DEM-derived projected area exponent, $D_{a}$, and prefactor, $k_{a}$, of small nascent soot aggregates do not depend on soot volume fraction and can be used in tandem with mass-mobility measurements to estimate the primary particle size with high accuracy.

In summary, the latest advances in particle characterization and aerosol dynamics have revealed the detailed evolution of such combustion-generated particles enabling scalable synthesis of uniquely functional materials (biomagnetic nanofluids, carbon nanotubes, nanosilver etc.) unraveling new applications in catalysis, gas sensors and biomaterials. Such new products are brought to market largely by start-ups. Furthermore, hand-held and portable devices are built with such flame-made functional materials as, for example, with gas sensors for breath analysis.

Though still a lot needs to be learned, especially with respect to early particle formation, crystallinity and surface composition, enough is known to make an impact to the needs of the society for sustainable living, energy and quality of life. Frankly, we live at a great time for flame aerosol technology and innovation in connection to material synthesis. The capacity of combustion to form multicomponent materials down to the atomic and nano level (mixed ceramics and alloys) cannot be matched by conventional technologies. Close interaction of combustion aerosol scientists with those advancing material applications in diverse fields of science and engineering will facilitate the unfolding of the potential and appreciation of combustion aerosol technology by the scientific community and the public at large.

\section{Acknowledgements}

The research leading to these results has received funding from the Swiss National Science Foundation (grant no. 200021_149144) and ETH Zurich. 


\section{References}

[1] M.D. Smooke, M.B. Long, B.C. Connelly, M.B. Colket, R.J. Hall, Combust. Flame 143 (2005) 613-628.

[2] S.B. Dworkin, Q. Zhang, M.J. Thomson, N.A. Slavinskaya, U. Riedel, Combust. Flame 158 (2011) 1682-1695.

[3] H. Wang, Proc. Combust. Inst. 33 (2011) 41-67.

[4] J. Camacho, C. Liu, C. Gu, H. Lin, Z. Huang, Q. Tang, X. You, C. Saggese, Y. Li, H. Jung, L. Deng, I. Wlokas, H. Wang, Combust. Flame 162 (2015) 3810-3822.

[5] R. Strobel, S.E. Pratsinis, J. Mater. Chem. 17 (2007) 4743-4756.

[6] K. Wegner, S.E. Pratsinis, Chem. Eng. Sci. 58 (2003) 4581-4589.

[7] T.D. Cabot, Beggar on Horseback. Boston, MA: Godine, 1979.

[8] G.D. Ulrich, Chem. Eng. News 62 (1984) 22-29.

[9] S.E. Pratsinis, S.V.R. Mastrangelo, Chem. Eng. Prog. 85 (1989) 62-66.

[10] G. Kühner, M. Voll, in: J.-B. Donnet, R.C. Bansal, M.-J. Wang (Eds.), Carbon Black, M. Dekker, New York, 1993, p. 1 .

[11] D.E. Rosner, Chem. Eng. Educ. 31 (1997) 228-235.

[12] S.E. Pratsinis, in: D.S. Ensor (Ed.), Aerosol Science and Technology: History and Reviews, RTI Press, Research Triangle Park, North Carolina, 2011, p. 475.

[13] G.D. Ulrich, Combust. Sci. Technol. 4 (1971) 47-57.

[14] M. Formenti, F. Juillet, P. Meriaudeau, S.J. Teichner, P. Vergnon, J. Colloid Interface Sci. 39 (1972) 79-89.

[15] M.S. Wooldridge, Prog. Energy Combust. Sci. 24 (1998) 63-87.

[16] S.E. Pratsinis, Prog. Energy Combust. Sci. 24 (1998) 197-219.

[17] P. Roth, Proc. Combust. Inst. 31 (2007) 1773-1788.

[18] M. Kraft, Kona Powder Part. J. 23 (2005) 18-35.

[19] B. Buesser, S.E. Pratsinis, Annu. Rev. Chem. Biomol. Eng. 3 (2012) 103-127.

[20] W.Y. Teoh, R. Amal, L. Mädler, Nanoscale 2 (2010) 1324-1347.

[21] R. Strobel, A. Baiker, S.E. Pratsinis, Adv. Powder Technol. 17 (2006) 457-480.

[22] B. Schimmoeller, S.E. Pratsinis, A. Baiker, ChemCatCem 3 (2011) 1234-1256.

[23] R. Koirala, S.E. Pratsinis, A. Baiker, Chem. Soc. Rev. 45 (2016) 3053-3068.

[24] M. Righettoni, A. Amann, S.E. Pratsinis, Mater. Today 18 (2015) 163-171.

[25] T. Dreier, C. Schulz, Powder Technol. 287 (2016) 226-238.

[26] S.E. Pratsinis, AIChE J. 56 (2010) 3028-3035. 
[27] A. Schätz, R.N. Grass, W.J. Stark, O. Reiser, Chem. Eur. J. 14 (2008) 8262-8266.

[28] M. Righettoni, A. Tricoli, S.E. Pratsinis, Anal. Chem. 82 (2010) 3581-3587.

[29] R. Strobel, L. Mädler, M. Piacentini, M. Maciejewski, A. Baiker, S.E. Pratsinis, Chem. Mater. 18 (2006) $2532-2537$.

[30] http://www.nano-c.com/.

[31] http://www.heiq.com/.

[32] http://www.turbobeads.com/.

[33] E.M. Schneider, M. Zeltner, V. Zlateski, R.N. Grass, W.J. Stark, Chem. Commun. 52 (2016) 938-941.

[34] R. Mueller, L. Mädler, S.E. Pratsinis, Chem. Eng. Sci. 58 (2003) 1969-1976.

[35] B. Thiebaut, Platinum Metals Rev. 55 (2011) 149-151.

[36] K. Wegner, B. Schimmoeller, B. Thiebaut, C. Fernandez, T.N. Rao, Kona Powder Part. J. 29 (2011) 251-265.

[37] M.C. Heine, S.E. Pratsinis, Ind. Eng. Chem. Res. 44 (2005) 6222-6232.

[38] C.D. Rosebrock, T. Wriedt, L. Mädler, K. Wegner, AIChE J. 62 (2016) 381-391.

[39] A.J. Gröhn, S.E. Pratsinis, A. Sanchez-Ferrer, R. Mezzenga, K. Wegner, Ind. Eng. Chem. Res. 53 (2014) 1073410742 .

[40] http://www.nanograde.com/.

[41] J.M. Rowell, J. Sci. Am. 255 (1986) 146-157.

[42] J.B. MacChesney, P.B. O’Connor, H.M. Presby, Proc. IEEE, 62 (1974) 1280-1281.

[43] K.S. Kim, S.E. Pratsinis, AIChE J. 34 (1988) 912-921.

[44] W. Koch, S.K. Friedlander, J. Colloid Interface Sci. 140 (1990) 419-427.

[45] R. Mueller, H.K. Kammler, S.E. Pratsinis, A. Vital, G. Beaucage, P. Burtscher, Powder Technol. 140 (2004) $40-48$.

[46] W. Zhu, S.E. Pratsinis, in: G.M. Chow, K.E. Gonsalves (Eds.), Nanotechnology, ACS Symp. Series, 1966, p. 64.

[47] F.E. Kruis, K.A. Kusters, S.E. Pratsinis, B. Scarlett, Aerosol Sci. Technol. 19 (1993) 514-526.

[48] S.E. Pratsinis, W.H. Zhu, S. Vemury, Powder Technol. 86 (1996) 87-93.

[49] T. Johannessen, S.E. Pratsinis, H. Livbjerg, Powder Technol. 118 (2001) 242-250.

[50] J. Hyeon-Lee, G. Beaucage, S.E. Pratsinis, S. Vemury, Langmuir 14 (1998) 5751-5756.

[51] E.J. Mezey, in: C.F. Palmer, J.H. Oxley, J.M. Blocher (Eds.), Vapor Deposition, John Wiley and Sons, New York, 1966, p. 423.

[52] Y. Xiong, M.K. Akhtar, S.E. Pratsinis, J. Aerosol Sci. 24 (1993) 301-313.

[53] S. Tsantilis, S.E. Pratsinis, Langmuir 20 (2004) 5933-5939.

[54] R.A. Dobbins, C.M. Megaridis, Langmuir 3 (1987) 254-259.

[55] O.I. Arabi-Katbi, S.E. Pratsinis, P.W. Morrison Jr., C.M. Megaridis, Combust. Flame 124 (2001) 560-572. 
[56] H.K. Kammler, G. Beaucage, D.J. Kohls, N. Agashe, J. Ilavsky, J. Appl. Phys. 97 (2005) 054309.

[57] S. Tsantilis, H.K. Kammler, S.E. Pratsinis, Chem. Eng. Sci. 57 (2002) 2139-2156.

[58] L. Mädler, H.K. Kammler, R. Mueller, S.E. Pratsinis, J. Aerosol Sci. 33 (2002) 369-389.

[59] B.S. Marshall, I. Telford, R. Wood, Analyst 96 (1971) 569-578.

[60] M. Sokolowski, M. Sokolawska, A. Michalski, B. Gokieli, J. Aerosol Sci. 8 (1977) 219-229.

[61] D.J. Lewis, J. Am. Ceram. Soc. 74 (1991) 2410-2413.

[62] C.R. Bickmore, K.F. Waldner, D.R. Treadwell, R.M. Laine, J. Am. Ceram. Soc. 79 (1996) 1419-1423.

[63] L. Mädler, S.E. Pratsinis, J. Amer. Ceram. Soc. 85 (2002) 1713-1718.

[64] Y. Xiong, S.E. Pratsinis, J. Aerosol Sci. 24 (1993) 283-300.

[65] S. Vemury, S.E. Pratsinis, J. Am. Ceram. Soc. 78 (1995) 2984-2992.

[66] R. Shirley, M. Kraft, O.R. Inderwildi, Phys. Rev. B 81 (2010) 075111.

[67] M.L. Eggersdorfer, S.E. Pratsinis, AIChE J. 59 (2013) 1118-1126.

[68] B. Buesser, A.J. Gröhn, S.E. Pratsinis, J. Phys. Chem. C 115 (2011) 11030-11035.

[69] E. Goudeli, S.E. Pratsinis, AIChE J. 62 (2016) 589-598.

[70] A. Violi, G.A. Voth, A.F. Sarofim, Proc. Combust. Inst. 30 (2005) 1343-1351.

[71] M.L. Eggersdorfer, A.J. Gröhn, C.M. Sorensen, P.H. McMurry, S.E. Pratsinis, J. Colloid Interface Sci. 387 (2012) 12-23.

[72] R. Weis, Washington Post, May 2, 2008, p. A04.

[73] G.A. Sotiriou, S.E. Pratsinis, Environ. Sci. Technol. 44 (2010) 5649-5654.

[74] E. Demou, W.J. Stark, S. Hellweg, Ann. Occup. Hyg. 53 (2009) 829-838.

[75] N. Barsan, U. Weimar, J. Electroceram. 7 (2001) 143-167.

[76] T. Sahm, L. Mädler, A. Gurlo, N. Barsan, S.E. Pratsinis, U. Weimar, Sens. Actuators, B 98 (2004) 148-153.

[77] Y. Liu, J. Dong, P.J. Hesketh, M. Liu, J. Mater. Chem. 15 (2005) 2316-2320.

[78] A. Teleki, S.E. Pratsinis, K. Kalyanasundaram, P.I. Gouma, Sens. Actuators, B 119 (2006) 683-690.

[79] L. Mädler, A. Roessler, S.E. Pratsinis, T. Sahm, A. Gurlo, N. Barsan, U. Weimar, Sens. Actuators, B 114 (2006) 283-295.

[80] A. Tricoli, M. Graf, F. Mayer, S. Kühne, A. Hierlemann, S.E. Pratsinis, Adv. Mater. 20 (2008) 3005-3010.

[81] S. Kühne, M. Graf, A. Tricoli, F. Mayer, S.E. Pratsinis, A. Hierlemann, J. Micromech. Microeng. 18 (2008) 035040.

[82] A.T. Güntner, M. Righettoni, S.E. Pratsinis, Sens. Actuators, B 223 (2016) 266-273.

[83] A.T. Güntner, N.J. Pineau, D. Chie, F. Krumeich, S.E. Pratsinis, J. Mater. Chem. B 4 (2016) 5358-5366.

[84] M. Righettoni, A. Tricoli, S. Gass, A. Schmid, A. Amann, S.E. Pratsinis, Anal. Chim. Acta 738 (2012) 69-75. 
[85] M. Righettoni, A. Schmid, A. Amann, S.E. Pratsinis, J. Breath Res. 7 (2013) 037110.

[86] M. Righettoni, A. Ragnoni, A.T. Güntner, C. Loccioni, S.E. Pratsinis, T.H. Risby, J. Breath Res. 9 (2015) 047101.

[87] A.T. Güntner, V. Koren, K. Chikkadi, M. Righettoni, S.E. Pratsinis, ACS Sensors 1 (2016) 528-535.

[88] G.A. Sotiriou, C.O. Blattmann, S.E. Pratsinis, Adv. Functional Mater. 23 (2013) 34-41.

[89] C.O. Blattmann, G.A. Sotiriou, S.E. Pratsinis, Nanotechnology 26 (2015) 125601.

[90] P.T. Spicer, C. Artelt, S. Sanders, S.E. Pratsinis, J. Aerosol Sci. 29 (1998) 647-659.

[91] H.K. Kammler, R. Mueller, O. Senn, S.E. Pratsinis, AIChE J. 47 (2001) 1533-1543.

[92] H.K. Kammler, S.E. Pratsinis, J. Mater. Res. 18 (2003) 2670-2676.

[93] E.K. Athanassiou, R.N. Grass, W.J. Stark, Nanotechnology 17 (2006) 1668-1673.

[94] R.N. Grass, E.K. Athanassiou, W.J. Stark, Angew. Chem. Int. Ed. 46 (2007) 4909-4912.

[95] I.K. Herrmann, R.N. Grass, D. Mazunin, W.J. Stark, Chem. Mater. 21 (2009) 3275-3281.

[96] T.J. Patey, R. Büchel, S.H. Ng, F. Krumeich, S.E. Pratsinis, P. Novak, J. Power Sources 189 (2009) 149-154.

[97] O. Waser, R. Büchel, A. Hintennach, P. Novak, S.E. Pratsinis, J. Aerosol Sci. 42 (2011) 657-667.

[98] F.O. Ernst, R. Büchel, R. Strobel, S.E. Pratsinis, Chem. Mater. 20 (2008) 2117-2123.

[99] I.D. Choi, H. Lee, Y.B. Shim, D. Lee, Langmuir 26 (2010) 11212-11216.

[100] J.M. Roller, R. Maric, J. Therm. Spray Technol. 24 (2015) 1529-1541.

[101] H. Lee, T.J. Kim, C. Li, I.D. Choi, Y.T. Kim, Z. Coker, T.Y Choi, D. Lee, Int. J. Hydrogen Energy 39 (2014) 14416-14420.

[102] M. Schenk, S. Lieb, H. Vieker, A. Beyer, A. Gölzhäuser, H. Wang, K. Kohse-Höinghaus, Proc. Combust. Inst. 35 (2015) $1879-1886$.

[103] S.E. Pratsinis, P.T. Spicer, Chem. Eng. Sci. 53 (1998) 1861-1868.

[104] D. Thompsett, in: G. Hoogers, Fuel Cell Technology Handbook, CRC Press, Florida, 2003.

[105] A. Star, V. Joshi, S. Skarupo, D. Thomas, J.C.P. Gabriel, J. Phys. Chem. B 110 (2006) 21014-21020.

[106] R.A. Dobbins, Aerosol Sci. Technol. 41 (2007) 485-496.

[107] C.M. Megaridis, R.A. Dobbins, Combust. Sci. Technol. 66 (1989) 1-16.

[108] A.D. Abid, N. Heinz, E.D. Tolmachoff, D.J. Phares, C.S. Campbell, H. Wang, Combust. Flame 154 (2008) 775 788.

[109] A. Neer, U.O. Koylu, Combust. Flame 146 (2006) 142-154.

[110] P. Pedata, T. Stoeger, R. Zimmermann, A. Peters, G. Oberdörster, A. D’Anna, Part. Fibre Toxicol. 12 (2015) 1-4.

[111] J. Rissler, E. Swietlicki, A. Bengtsson, C. Boman, J. Pagels, T. Sandström, A. Blomberg, J. Löndahl, J. Aerosol Sci. 48 (2012) 18-33. 
[112] R.A. Dobbins, Combust. Flame 130 (2002) 204-214.

[113] P. Mitchell, M. Frenklach, Phys. Rev. E 67 (2003) 061407.

[114] P.T.A. Reilly, R.A. Gieray, W.B. Whitten, J.M. Ramsey, Combust. Flame 122 (2000) 90-104.

[115] M.M. Maricq, S.J. Harris, J.J. Szente, Combust. Flame 132 (2003) 328-342.

[116] H. Oltmann, J. Reimann, S. Will, Appl. Phys. B 106 (2012) 171-183.

[117] M. Schenk, S. Lieb, H. Vieker, A. Beyer, A. Gölzhäuser, H. Wang, K. Kohse-Höinghaus, PhysChemPhys 14 (2013) 3248-3254.

[118] M.L. Eggersdorfer, S.E. Pratsinis, Adv. Powder Technol. 25 (2014) 71-90.

[119] J.S. Bhatt, R.P. Lindstedt, Proc. Combust. Inst. 32 (2009) 713-720.

[120] R.P. Lindstedt, B.B.O. Waldheim, Proc. Combust. Inst. 34 (2013) 1861-1868.

[121] C. Saggese, S. Ferrario, J. Camacho, A. Cuoci, A. Frassoldati, E. Ranzi, H. Wang, T. Faravelli, Combust. Flame 162 (2015) 3356-3369.

[122] E.K.Y. Yapp, D. Chen, J. Akroyd, S. Mosbach, M. Kraft, J. Camacho, H. Wang, Combust. Flame 162 (2015) $2569-2581$.

[123] P. Mitchell, Monte Carlo Simulation of Soot Aggregation with Simultaneous Surface Growth, PhD thesis, University of California, Berkeley, U.S.A., 2001.

[124] N. Morgan, M. Kraft, M. Balthasar, D. Wong, M. Frenklach, P. Mitchell, Proc. Combust. Inst. 31 (2007) 693-700.

[125] E. Goudeli, M.L. Eggersdorfer, S.E. Pratsinis, Langmuir 31 (2015) 1320-1327.

[126] A. Kazakov, H. Wang, M. Frenklach, Combust. Flame 100 (1995) 111-120.

[127] J. Appel, H. Bockhorn, M. Frenklach, Combust. Flame 121 (2000) 122-136.

[128] M. Frenklach, D.W. Clary, W.C. Gardiner, S.E. Stein, Proc. Combust. Inst. 20 (1985) 887-901.

[129] M. Frenklach, H. Wang, Proc. Combust. Inst. 23 (1991) 1559-1566.

[130] J. Lahaye, F. Ehrburger-Dolle, Carbon 32 (1994) 1319-1324.

[131] S.K. Friedlander, Smoke, Dust, and Haze: Fundamentals of Aerosol Dynamics. 2nd ed., Oxford University Press, New York, 2000.

[132] M. Ben Ohoud, F. Obrecht, L. Gatineau, P. Levitz, H. Van Damme, J. Colloid Interface Sci. 124 (1988) $156-161$.

[133] K. Park, F. Cao, D.B. Kittelson, P.H. McMurry, Environ. Sci. Technol. 37 (2003) 557-583.

[134] P. Meakin, B. Donn, G.W. Mulholland, Langmuir 5 (1989) 510-518.

[135] R.C. Ball, R. Jullien, J. Phys. Lett. 45 (1984) L1031-L1035.

[136] C.M. Sorensen, Aerosol Sci. Technol. 45 (2011) 765-779.

[137] P. Pfeifer, D. Avnir, D. Farin, Surf. Sci. 126 (1983) 569-572. 
[138] A.I. Medalia, J. Colloid Interface Sci. 24 (1967) 393-404.

[139] M.C. Heine, S.E. Pratsinis, Langmuir 23 (2007) 9882-9890.

[140] C. Gu, H. Lin, J. Camacho, B. Lin, C. Shao, R. Li, H. Gu, B. Guan, Z. Huang, H. Wang, Combust. Flame 165 (2016) $177-187$.

[141] P.B. Sunderland, G.M. Faeth, Combust. Flame 105 (1996) 132-146.

[142] S. Kumar, D. Ramkrishna, Chem. Eng. Sci. 52 (1997) 4659-4679.

[143] P.T. Spicer, O. Chaoul, S. Tsantilis, S.E. Pratsinis, J. Aerosol Sci. 33 (2002) 17-34.

[144] N.A. Fuchs, The mechanics of aerosols, Dover Publications Inc., New York, 1964.

[145] J. Pich, S.K. Friedlander, F.S. Lai, J. Aerosol Sci. 1 (1970) 115-126.

[146] S.H. Park, K.W. Lee, M. Shimada, K. Okuyama, J. Aerosol Sci. 32 (2001) 187-197.

[147] D.R. Warren, J.H. Seinfeld, J. Colloid Interface Sci. 105 (1985) 136-142.

[148] M. Kolb, R. Jullien, J. Phys. Let. 45 (1984) 977-981.

[149] S.E. Pratsinis, J. Colloid Interface Sci. 124 (1988) 416-427.

[150] J.D. Landgrebe, S.E. Pratsinis, Ind. Eng. Chem. Res. 28 (1989) 1474-1481.

[151] A. Kazakov, M. Frenklach, Combust. Flame 112 (1998), 270-274.

[152] S. Lieb, Experimental Investigation of Nascent Soot Physical Properties and The Influence on Particle Morphology and Growth, PhD thesis, University of Southern California, U.S.A. 2014.

[153] C. Lee, T.A. Kramer, Adv. Colloid Interface Sci. 112 (2004) 49-57.

[154] M.L. Eggersdorfer, D. Kadau, H.J. Herrmann, S.E. Pratsinis, J. Aerosol Sci. 46 (2012) 7-19.

[155] A.D. Abid, J. Camacho, D.A. Sheen, H. Wang, Combust. Flame 156 (2009) 1862-1870.

[156] C. Oh, C.M. Sorensen, J. Colloid Interface Sci. 193 (1997) 17-25.

[157] M. Bönig, C. Feldermann, H. Jander, B. Luers, G. Rudolph, H.G. Wagner, Proc. Combust Inst. 23 (1990) 15811587.

[158] R. Ghazi, H. Tjong, A. Soewono, S.N. Rogak, J.S. Olfert, Aerosol Sci. Technol. 47 (2013) 395-405.

[159] M.L. Eggersdorfer, D. Kadau, H.J. Herrmann, S.E. Pratsinis, Langmuir 27 (2011) 6358-6367.

[160] M. Balthasar, M. Frenklach, Combust. Flame 140 (2005) 130-145.

[161] S. Tsantilis, S.E. Pratsinis, J. Aerosol Sci. 35 (2004) 405-420. 